\title{
The Ca II triplet lines as diagnostics of luminosity, metallicity and chromospheric activity in cool stars ${ }^{\star}$
}

\author{
S.V. Mallik \\ Indian Institute of Astrophysics, Bangalore 560034, India
}

Received July 18; accepted November 27, 1996

\begin{abstract}
CCD spectra of the infrared triplet lines of ionized calcium at $\lambda \lambda 8498,8542,8662$ have been obtained at a spectral resolution of $0.4 \AA$ in 146 stars brighter than $V=+7.0$ spanning a range in spectral types from $\mathrm{F} 7$ to M4 of all luminosity classes and a range in metallicity $[\mathrm{Fe} / \mathrm{H}]$ from -3.0 to +1.1 . These have been analysed to investigate the dependence of the Ca II triplet strengths on stellar parameters like luminosity, temperature and metallicity. A detailed study reveals a strong dependence on luminosity, much stronger for metal rich stars than for the metal poor ones and a milder dependence on metallicity, although much more conspicuous in supergiants than in dwarfs. All these correlations are found to be non-linear over the parameter space covered. The present study also shows chromospheric activity to be an important phenomenon affecting the strength and the shape of the line profiles. Stars of similar luminosity and metallicity have varying Ca II line depths owing to varying chromospheric emission filling in their Ca II absorption. The Ca II triplet strength is thus observed to be a triparametric discriminant in cool stars.
\end{abstract}

Key words: stars: late-type — stars: chromospheres galaxies: stellar content

\section{Introduction}

The Ca II resonance lines $H(\lambda 3968)$ and $K(\lambda 3933)$ and the $\mathrm{Mg}$ II resonance lines $h(\lambda 2803)$ and $k(\lambda 2796)$ have been traditionally used as diagnostics of luminosity and chromospheric activity in cool stars (Linsky 1980 and the references therein, Cassinelli \& MacGregor 1986). Other useful indicators of chromospheric gas include the Ly $\alpha$ and the Balmer lines of H I, the infrared triplet lines $(\lambda \lambda 8498$,

Send offprint requests to: S.V. Mallik

* Tables 1 and 2 are available in electronic form only at the CDS via anonymous ftp to cdsarc.u-strasbg.fr (130.79.128.5) or via http://cdsweb.u-strasbg.fr/Abstract.html
$8542,8662)$ of $\mathrm{Ca}$ II and the He I lines at $\lambda 5876$ line and $\lambda 10830$. The ultraviolet lines have a limitation because of the low flux of cool stars in this part of the spectral region, making it difficult to obtain spectra with high signal-tonoise ratio. It is worth exploring lines, better placed in the spectrum, that are sensitive to luminosity and other parameters. The Ca II triplet lines at $\lambda \lambda 8498,8542,8662$ are ideal candidates for studying their sensitivity to various stellar parameters in cool stars. These lines are due to transitions between the upper $4 \mathrm{p}^{2} \mathrm{P}_{1 / 2,3 / 2}$ levels and the lower metastable $3 \mathrm{~d}{ }^{2} \mathrm{D}_{3 / 2,5 / 2}$ levels. The same upper levels connect to the ground state $4 \mathrm{~s}^{2} \mathrm{~S}_{1 / 2}$ to give rise to the $\mathrm{Ca}$ II $\mathrm{H}$ and $\mathrm{K}$ lines. Although the Ca II triplet lines have the lower levels populated radiatively and are not collisionally controlled like the Ca II H and K lines, they have been identified as very good luminosity probes. These lines are strong, hence easy to observe even in faint stars. They are relatively free from blends and are not contaminated much by the telluric lines. On the contrary, the $\mathrm{Na} I$ lines at $\lambda \lambda 8183,8195$, which have also been used as luminosity probes in cool stars, have several atmospheric absorption lines in their vicinity (Jones et al. 1984; Carter et al. 1986; Alloin \& Bica 1989; Zhou 1991).

With the advent of the CCDs which are especially sensitive to the red, there has been a spurt of studies of the Ca II triplet lines in both stars and galaxies, to investigate, in particular, the behaviour of their strengths as a function of surface gravity (which is directly proportional to luminosity), metallicity and temperature and to use this information in stellar population synthesis (Jones et al. 1984 (JAJ); Carter et al. 1986; Alloin \& Bica 1989; Diaz et al. 1989 (DTT) and Zhou 1991). JAJ observed a sample of 62 stars covering a range in spectral types from early B to mid-M and over 4 orders of surface gravity and a factor of 10 in metallicity. Examination of their data led JAJ to suggest a single-valued relation between the Ca II equivalent width (EQW) and the surface gravity for all stars in the spectral range $\mathrm{F}$ through mid-M. DTT in their comprehensive study of 106 stars over the spectral range F6 to M0 and of all luminosity classes found that the Ca II lines exhibited a 
biparametric behaviour; there is almost no influence of $T_{\text {eff }}$ but there is dependence on gravity and metallicity. They concluded that in the high-metallicity range, the Ca II triplet lines depend on surface gravity only while in the low-metallicity range they depend on metallicity as well.

There has also been a number of theoretical studies to understand the effects of stellar parameters on the Ca II triplet strengths (Smith \& Drake 1987, 1990; ErdelyiMendes \& Barbuy 1991; Jorgenson et al. 1992 (JCJ)). JCJ carried out detailed non-LTE calculations of the Ca II EQWs over an extended span of parameters and concluded that although the correlation between the Ca II EQW and $\log g$ is straightforward, the dependence on $T_{\text {eff }}$ and metallicity is more complex. Their theoretical work has shown that the line EQWs depend on the response of the stellar atmospheric structure to changes in these parameters and more importantly, the response to a change of a given parameter is different for different parameter intervals considered of the other parameters. Because of this complex behaviour of the lines, the question of whether the nucleus of a galaxy or any composite stellar system is dwarf enriched, for example, and/or metal rich has remained an open one.

In order to further explore the dependence of the Ca II triplet EQWs on these stellar parameters, we had, in our previous study (Mallik 1994) observed the first two lines of the triplet, namely, the $8498 \AA$ and the $8542 \AA$ lines in 91 stars in the spectral range F8 - M4 of all luminosity classes and over a range of metallicities $[\mathrm{Fe} / \mathrm{H}]$ from -0.65 to +0.60 . A detailed analysis of these observations confirmed that the sum of the equivalent widths (EQWs) of $\lambda 8498$ and $\lambda 8542$ strongly correlates with surface gravity and also depends upon metallicity. As JCJ have emphasised, it is very important for a proper understanding of the behaviour of the Ca II triplet EQWs that a sufficiently large range in the values of parameters be covered. We have in our current analysis enlarged the earlier sample to 146 stars including more dwarfs, subgiants and many more number of metal poor stars; some with a much lower metal content. In particular, our sample now includes a few superluminous supergiants (of luminosity 0Ia) which turn out to be crucial in deriving the behaviour of the Ca II EQWs. Also, since the $\lambda 8662$ line lies in a comparatively clean region of the spectrum with very few blends and since it is possible to determine its EQW accurately, observations of the $\lambda 8662$ line have also therefore been obtained for the entire sample. Section 2 describes the observations and data reduction. The analysis of the Ca II triplet observations is discussed in Sect. 3. Section 4 discusses chromospheric activity as an important phenomenon influencing the line depth and hence the EQW of the Ca II triplet line profiles. Conclusions are given in Sect. 5 .

\section{Observations and data reduction}

A large number of cool stars have been chosen from the Bright Star Catalogue (Hoffleit 1982) and the $[\mathrm{Fe} / \mathrm{H}]$ catalogue of Cayrel de Strobel et al. (1992) brighter than $V=+7.0$ and ranging in spectral types from F5 to M4, of all luminosity types. Observations were carried out at the Vainu Bappu Observatory, Kavalur with the coude echelle spectrograph at the $102 \mathrm{~cm}$ telescope using a $384 \times 576 \mathrm{CCD}$ detector, each pixel of $23 \mu$ square size. The spectrograph comprised of a $79 \mathrm{lmm}^{-1}$ echelle grating blazed at $6746 \AA$ in the 34 th order, a $150 \mathrm{lmm}^{-1}$ cross dispersion grating blazed at $8000 \AA$ in the 1st order and a $25 \mathrm{~cm}$ camera. This configuration gave a dispersion of about $9 \AA \mathrm{mm}^{-1}$ and with the slit width used, a spectral resolution of about $0.4 \AA(\lambda / \Delta \lambda \simeq 20000)$ in the 26 th order where the Ca II triplet lines lie. The $8498 \AA$, $8542 \AA$ \& $8662 \AA$ lines have been observed in 146 stars, spanning 5 orders of magnitude in $g$, a factor of $10^{4}$ in metallicity, $[\mathrm{Fe} / \mathrm{H}]$ ranging from -3.0 to +1.01 . But note however that only one star has $[\mathrm{Fe} / \mathrm{H}]=-3.0$; the rest lie between $[\mathrm{Fe} / \mathrm{H}]=-1.6$ and +1.01 , spanning a range of metallicity over a factor of 400 .

Because of the fairly high spectral resolution, all three lines could not be observed together in the same spectral interval i.e. in the same order of the echelle spectrum. A different setting of the grating was required for the $\lambda 8662$ line and hence for each star the coverage required two frames each. Subject to the sky/seeing conditions, for stars fainter than $V=+5.0$, often more than one frame was required and then the frames were added to obtain a better signal-to-noise ratio. In most cases, the $\mathrm{S} / \mathrm{N}$ ratio was of 50 or higher. A Thorium-Argon hollow cathode lamp was used for line identification and a Xenon lamp was used as a flat field source. Sufficient number of bias, comparison and flat field frames were taken each night well spaced out in time in between the star frames.

Table 1 gives the list of the program stars, their spectral types and visual magnitudes in the first 5 columns. Columns 6, 7, 8 and 9 list the stellar parameters, namely, $\theta_{\text {eff }},(R-I), \log g$ and $[\mathrm{Fe} / \mathrm{H}]$ in that order, taken from the $[\mathrm{Fe} / \mathrm{H}]$ Catalogue of Cayrel de Strobel et al. (1992) and from the Bright Star Catalogue (Hoffleit 1982). $(R-I)$ is a very good indicator of temperature for cool stars. Here $\theta_{\text {eff }}=5040 / T_{\text {eff }}$. Since processing of elements eventually leads to $\mathrm{Fe}$ production, $[\mathrm{Fe} / \mathrm{H}]$ is taken as a metallicity index. The $[\mathrm{Fe} / \mathrm{H}]$ catalogue often gives more than one set of values of $\theta_{\text {eff }}, \log g$ and $[\mathrm{Fe} / \mathrm{H}]$ for a given star, based on different studies done of that star. Care was taken to choose the ones derived from the best quality data, i.e., data obtained at high resolution and/or in red/near-infrared and the ones based on fine analysis/spectrum synthesis. These are more reliable because the red region is less line-crowded than the blue, thus minimizing the effects of blanketing and allowing a better location of the continuum. The stars listed in Table 
1 include a distribution of dwarfs, subgiants, giants and supergiants with metallicities spread over a large enough range. Five stars have $[\mathrm{Fe} / \mathrm{H}]$ between -1.0 and $-3.0,22$ between -0.5 and -1.0 , and 43 between 0.0 and -0.5 ; 42 stars are metal rich with $[\mathrm{Fe} / \mathrm{H}]$ between +0.00 and +1.01 , while only 5 of them have $[\mathrm{Fe} / \mathrm{H}] \geq+0.50$. Because of the restriction of observations to stars with a magnitude brighter than $V=+7.0$, not many of the more metal poor ones $([\mathrm{Fe} / \mathrm{H}] \leq-1.6)$ could be included. Also, our sample does not contain a large number of metal rich (with $[\mathrm{Fe} / \mathrm{H}] \geq+0.50$ ) G, K and M stars. Since they span all ages, their number is heavily weighted towards older, metal poor populations. A G, K or M metal rich star is much rarer than a metal poor one. Similarly, it is more likely to find an A type (or hotter) star which is metal rich, than a cool star which is metal rich.

There are several stars in Table 1 for which $[\mathrm{Fe} / \mathrm{H}]$ and $\log g$ are not yet available from the literature. Wherever $\log g$ is not available, we have used the statistical values given in Allen (1973) and Zhou (1991). Table 1 also contains a few superluminous supergiants, i.e., of luminosity class 0 and 0 -Ia, also called hypergiants. Some of these and several more appear in "An Atlas of Spectra of the cooler stars" (Keenan \& McNeil 1976). According to this atlas, these stars are known to have $M_{V}$ as high as -8.5 to -9.0 , comparable to the most luminous stars in the LMC. Gravities for most of the superluminous supergiants could not be estimated from Allen (1973) or Zhou (1991). They were estimated from their basic stellar data in the following way. The effective temperatures were obtained from the temperature calibrations of Bohm-Vitense (1972), Flower (1977), Luck \& Bond (1980) and Bell \& Gustaffson (1989). The absolute visual magnitude $M_{V}$ for these stars were obtained from the MK- $M_{V}$ mapping tabulated for various spectral types and luminosities (Lang 1992). Bolometric corrections (B.C.) are from SchmidtKaler (1982). Once $T_{\text {eff }}$ and $M_{\text {bol }}$ are known, $R$, the stellar radius could be calculated:

$M_{\mathrm{bol}}=-2.5 \log L / L_{\odot}+4.64$

$M_{\mathrm{bol}}=42.26-5 \log R / R_{\odot}-10 \log T_{\text {eff }}$

where $M_{\mathrm{bol}}=M_{V}+$ B.C.

$M_{\mathrm{bol}, \odot}=4.64, T_{\mathrm{eff}, \odot}=5780 \mathrm{~K}, L_{\odot}=3.8510^{33} \mathrm{erg} \mathrm{s}^{-1}$.

Once $R$ is known, and the mass $M$ is assumed (from the location of the star on the HR diagram), $g$ can be calculated:

$g=G M / R^{2} ; g_{\odot}=G M_{\odot} / R_{\odot}^{2}$

$g / g_{\odot}=\frac{M / M_{\odot}}{\left(R / R_{\odot}\right)^{2}} \Rightarrow g=g_{\odot} M / R^{2}$

where $M$ and $R$ are in solar units; $g_{\odot}=2.73810^{4} \mathrm{~cm} \mathrm{~s}^{-1}$.
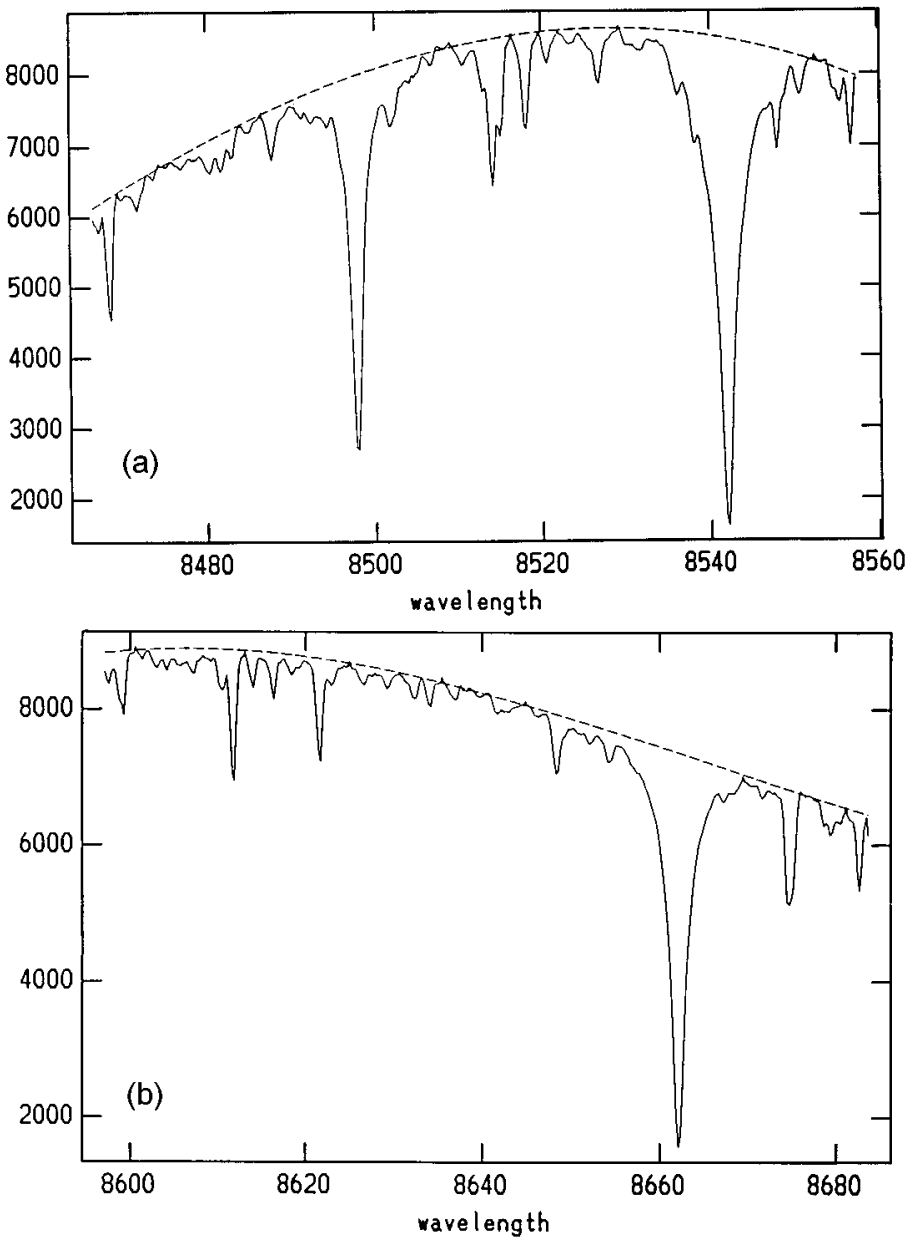

Fig. 1. A first order cubic spline fit to the continuum in $\alpha$ Boo: a) $\lambda \lambda 8498,8542$, b) $\lambda 8662$

The values of $\log g$ listed in Table 1 with asterix are the ones determined as above.

A part of the data reduction was carried out with the RESPECT software package due to Prabhu \& Anupama (1991) installed at the Vax 11/780 system at VBO. Here, the procedure of extracting one-dimensional spectra from the two dimensional CCD image follows the optimal extraction algorithm of Horne $(1986,1988)$. Since for each star, the $\lambda \lambda 8498,8542$ and the $\lambda 8662$ spectra were observed separately, the reduction of their data was done independently for the two spectra. The reduction procedure involved bias subtraction, flat field correction, wavelength calibration and normalisation by fitting a continuum. The bias was subtracted from the raw spectrum, then the spectrum was divided by the flat field image which accounted both for the pixel to pixel sensitivity difference of the detector and for the curved nature of the response function of the echelle spectrograph across each order. The wavelength scales for the observed spectra were derived using the absorption lines in the stellar spectrum itself, ensuring that the lines are of photospheric origin 
(with lower excitation potential $\geq 2 \mathrm{eV}$ ). The stellar features chosen in the neighbourhood of $\lambda \lambda 8498,8542$ are Fe I lines at $8468.418 \AA, 8471.744 \AA, 8514.082 \AA$, $8515.122 \AA$ and $8526.676 \AA$ and $\mathrm{a} \mathrm{Si}$ I line at $8556.797 \AA$. In the neighbourhood of $\lambda 8662$ are the Fe I lines at $8610.609 \AA, 8611.812 \AA, 8613.946 \AA, 8616.284 \AA$, $8621.618 \AA, 8674.756 \AA$ and $8688.642 \AA$ and the Si I lines at $8648.472 \AA$ and $8686.368 \AA$. The analysis of the Ca II triplet lines requires only the measurement of equivalent widths, so no flux calibration was done. Instead, each observed spectrum was divided by an estimated continuum and the EQWs were measured from the normalised spectra thus obtained. Rest of the data was reduced with the CCD reduction package IRAF (Image Reduction and Analysis Facility) developed in the UNIX operating environment and is available on a SUN workstation. Spectra of a few stars were reduced both with the RESPECT and the IRAF to see how well the EQWs determined in the two ways compare. The correlation was found to be good to within 5 per cent.

The EQW measurement depends most crucially upon the choice of the continuum. TiO bands appear in the vicinity of the Ca II features stretching from $8432 \AA$ to $8620 \AA$. The $\mathrm{TiO}$ bands with the triple bandheads at $\lambda \lambda 8432,8442$ and 8452 become very strong in stars with spectral types M4 and later, and tend to depress the continuum in the vicinity of the Ca II triplet lines which thus appear weaker. The uncertainty in the placement of the continuum, because of the $\mathrm{TiO}$ bands, can therefore give rise to serious uncertainties in the EQW measurements. In most of the previous studies where the 3 lines were observed simultaneously, owing to the lower resolution, the practice has been to choose two wavelength bands over the entire spectral range observed and to define the continuum by a straight line fit relative to the flux maxima near the chosen wavelength bands. Fitting a straight line continuum with local maxima at the bands chosen close to each of the Ca II triplet lines has the effect of eliminating the contribution from the wings of the Ca II lines which results in underestimating the EQW. On the other hand, fitting a continuum between the local maxima at the bands far to the right and left side of the Ca II line results in an overestimate of the EQW because of the inclusion of $\mathrm{TiO}$ bands especially for the cooler stars. Since the present observations were obtained at a fairly high spectral resolution and the three lines could not be observed together, the latter method for choosing the continuum could not be adopted. Instead, a few points were chosen consistently for spectra of all the stars, in the regions relatively free of spectral lines across the order containing the Ca II triplet lines. As a representative sample, Figs. 1a and b show respectively for $\lambda \lambda 8498,8542$ and for $\lambda 8662$ the continuum fitting obtained by a first order cubic spline fit over the chosen points. Care has to be taken to define the continuum relative to the same wavelength regions in stars of all spectral types so that it provides a consistent method for studying mixed stellar populations. From the data for stars observed more than once, the error in the determination of EQWs for each individual observation could be estimated and this turns out to be less than 10 per cent. M stars would give larger errors because of the presence of strong TiO bands. We have very few $\mathrm{M}$ stars on our list; in fact none later than M4 are there. However, for the early M stars where the $\mathrm{TiO}$ bands do start showing up, there was some ambiguity in the location of the continuum. Our experience with EQW measurements from over 300 spectra revealed that errors in EQWs arise mostly out of uncertainties in the continuum placing and could amount to $10 \%-20 \%$.

Columns 4, 5 and 6 of Table 2 list the EQWs of $\lambda 8498$, $\lambda 8542$ and $\lambda 8662$ respectively. Column 7 gives the sum of the 3 EQWs designated as CaT. The observations of DTT (1989), for example, involved the sum of the EQWs of $\lambda 8542$ and $\lambda 8662$ and not of all the three lines of the Ca II triplet. Theoretical analysis by JCJ (1992) also lists the sum of the two brighter lines. For comparing the present values with these, we have listed the sum of the observed EQWs of $\lambda 8542$ and $\lambda 8662$, denoted by $\mathrm{W}$ in Col. 8. For stars in common with DTT, the present values are lower roughly by 5 to $20 \%$ in 15 of them and $25-35 \%$ for 7 of them and for those in common with Zhou (1991), the present values are lower by an even larger factor. On the other hand, our $\lambda 8498$ measures of 14 stars in common with those of Anderson (1974) are higher than his and also our $\lambda 8542$ measures of 25 stars in common with Linsky et al. (1979) are higher than theirs. These differences are certainly due in part to the choice of the line windows for the EQW measurement of the 3 lines which indirectly has to do with the spectral resolution used in each study. The EQW measurements of the Ca II triplet observed by DTT at a spectral resolution of $3.5 \AA$ have been based on a choice of a line window of $30 \AA$. Zhou's (1991) observations were obtained at a spectral resolution of $2 \AA$ and the chosen line window was $20 \AA$. As a consequence, several features of Ti I, Fe I, Si I, Ni I and CN either blended with a Ca II triplet line or on either side of it have contributed to the resultant EQW. Figures $2 \mathrm{a}, \mathrm{b}$ and $\mathrm{c}$ show the $30 \AA$ region around the 3 lines respectively in $\alpha$ Boo as an example, with several lines identified. About 9 in the vicinity of $\lambda 8662,12$ around $\lambda 8542$ and about 13 features in the neighbourhood of $\lambda 8498$ have added to the measured EQWs. The line windows chosen in the present observations were much smaller, namely, $8492 \AA-8504 \AA$ for $\lambda 8498 ; 8535 \AA-8550 \AA$ for $\lambda 8542$ and $8656 \AA-8669 \AA$ for $\lambda 8662$. These therefore included less blended features that contribute to the EQWs of the Ca II triplet lines. The window for $\lambda 8498$ includes, besides small contributions of Fe I, Atm $\mathrm{H}_{2} \mathrm{O} \lambda 8493.796$, Ti I $\lambda 8494.44$, Ti I $\lambda 8496.075$ and Fe I $\lambda 8496.994$, the lines of Si I $\lambda \lambda 8501.553,8502.228$ and Ni I $\lambda 8501.803$. The window chosen for $\lambda 8542$ has Si I $\lambda 8536.163, \mathrm{Fe}$ I $\lambda 8538.021$, Ti I $\lambda 8548.079$ and to a smaller extent CN, Cr I $\lambda 8548.863$ and that for $\lambda 8662$ contains 

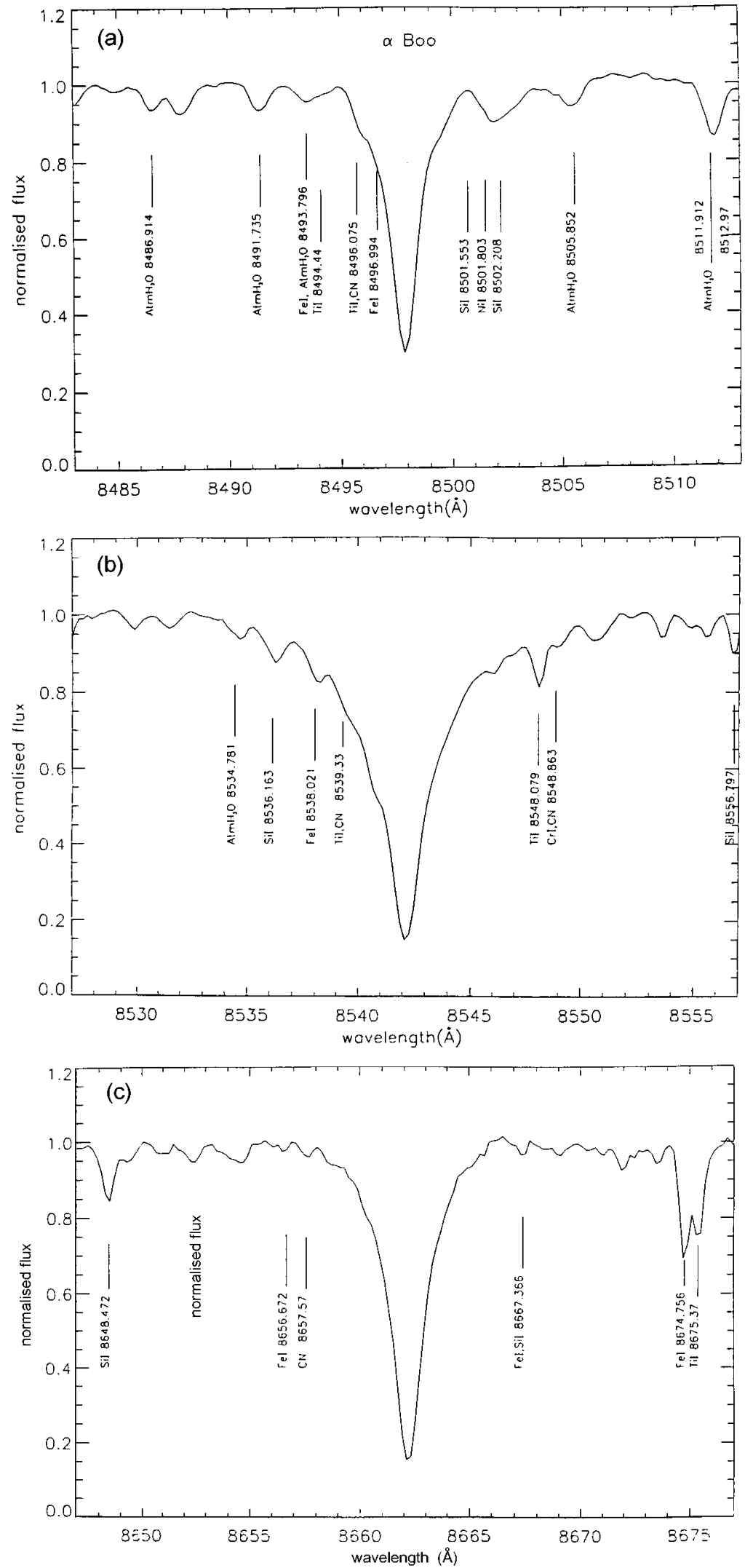

Fig. 2. $30 \AA$ normalised (divided by the continuum) spectral region in $\alpha$ Boo in the neighbourhood of: a) $\lambda 8498$, b) $\lambda 8542$, c) $\lambda 8662$ 
(a)
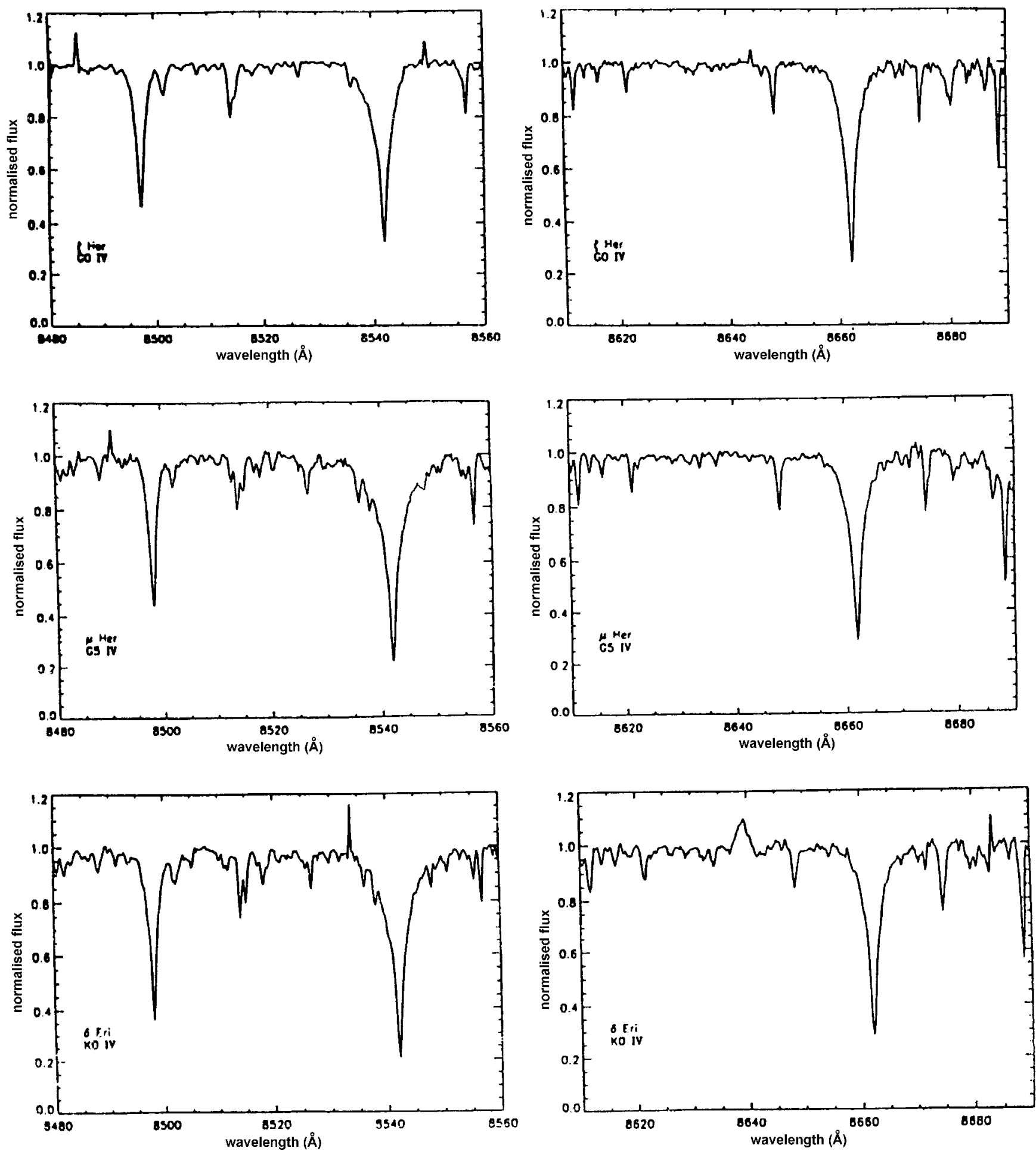

Fig. 3. Sample normalised spectra around the $\lambda \lambda 8498,8542,8662$ lines of Ca II for stars of a given luminosity over a range of spectral types: a) IV, b) II, c) Ib, d) 0-Ia 
(b)
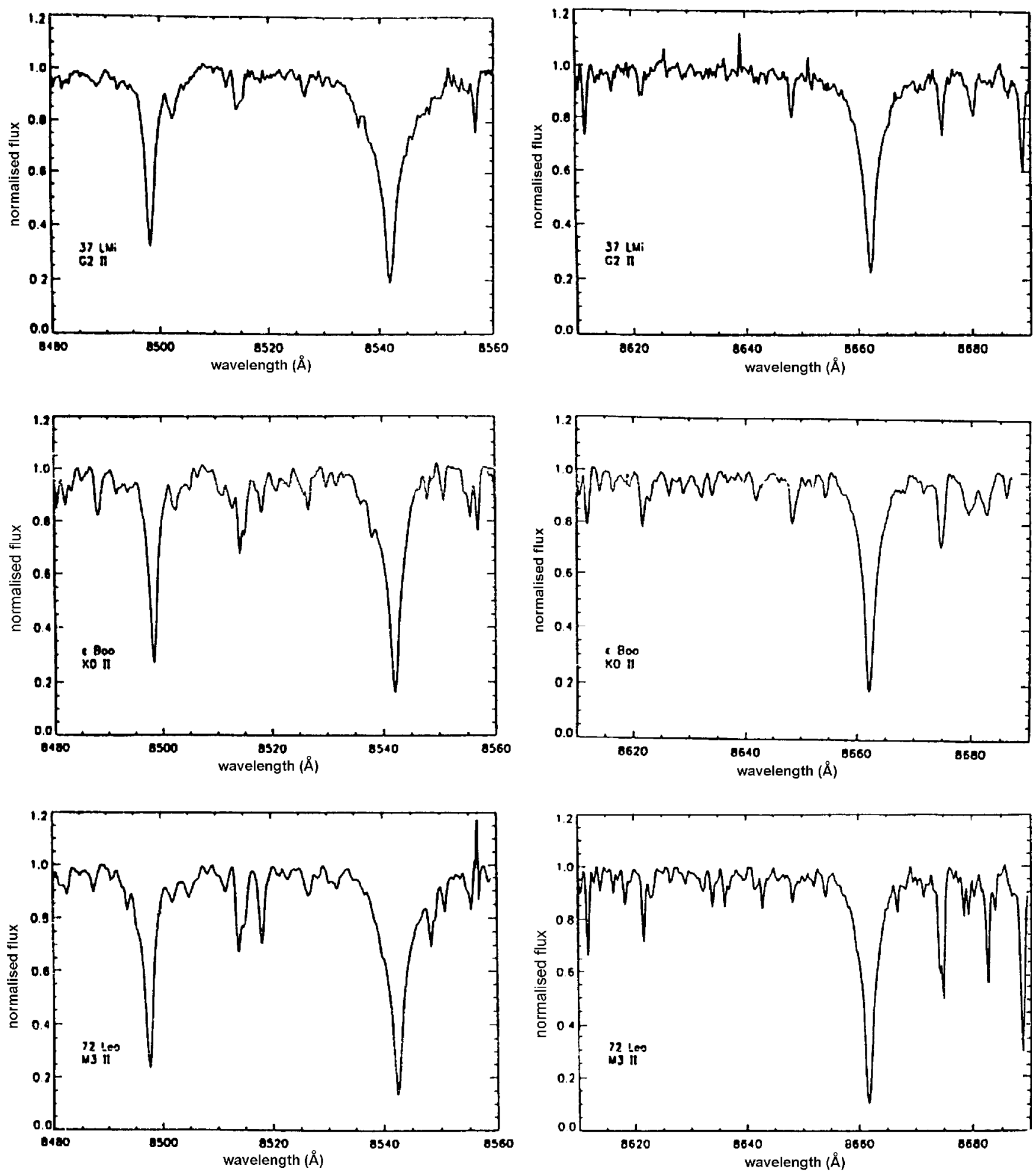

Fig. 3. continued 
(c)
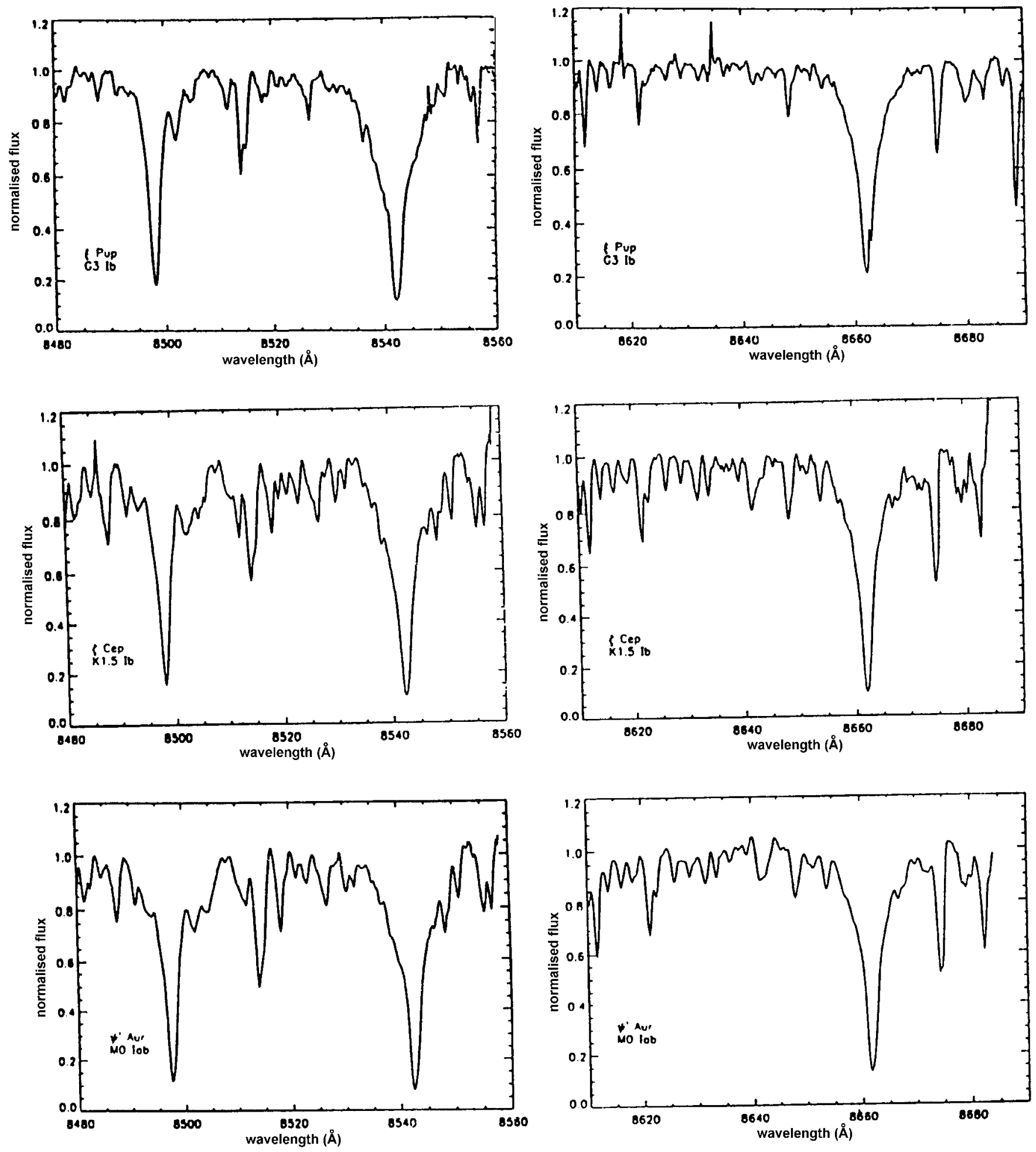

Fig. 3. continued 
(d)
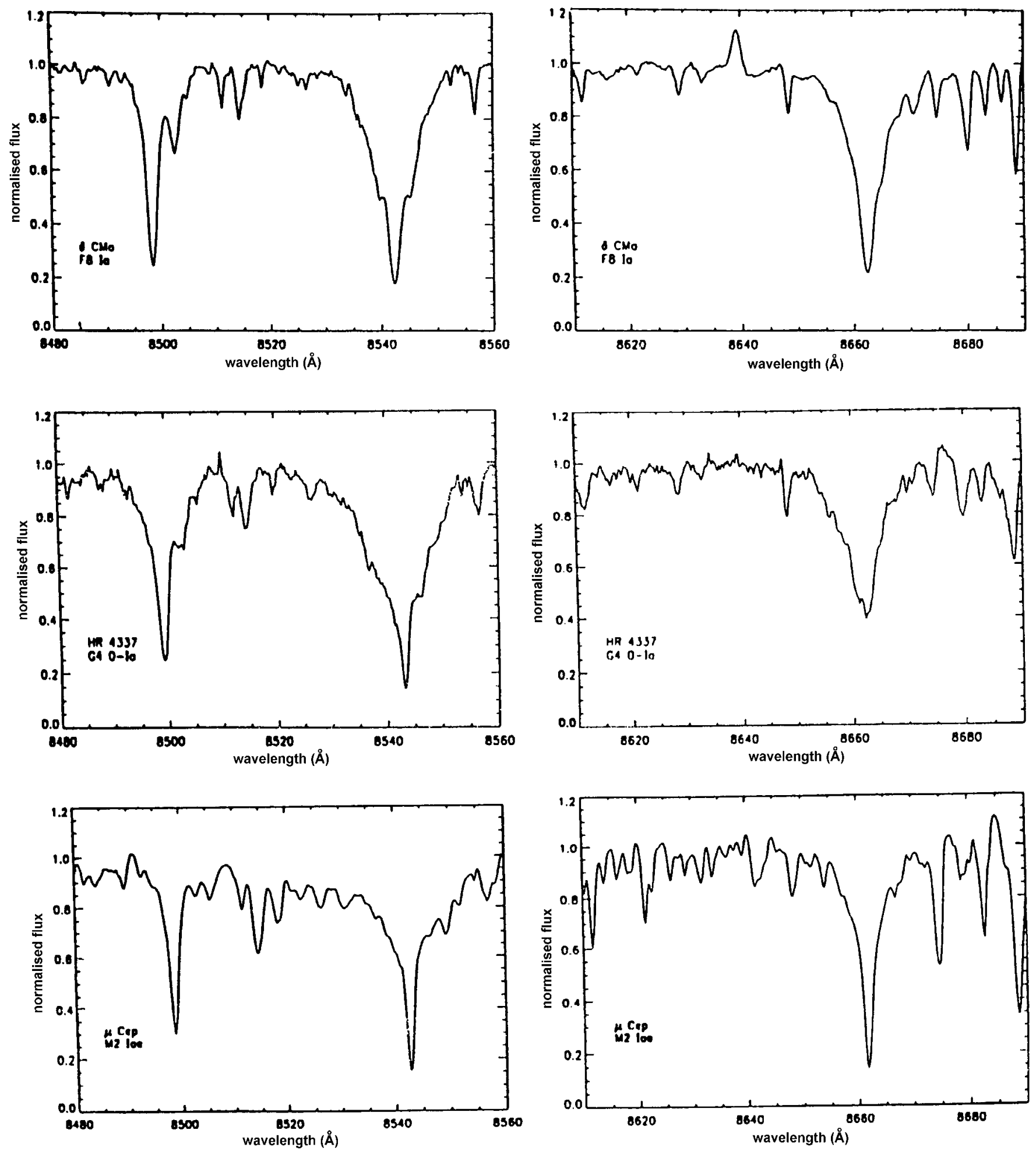

Fig. 3. continued 
Fe I $\lambda 8656.672, \mathrm{CN} \lambda 8657.57$ and Fe I, Si I $\lambda 8667.366$, none of which are strong. There is less contamination from the neighbourhood of the lines owing to the smaller windows chosen, hence lower EQWs. The observations of Linsky et al. (1979) and of Anderson (1974) are at a much higher spectral resolution $(0.14 \AA$ and $0.28 \AA$ respectively) than ours; perhaps the effect of blending is minimized. For 23 of our program stars in common with DTT, we remeasured the EQWs choosing the line window to be $30 \AA$. It turns out that the EQWs are larger, as expected, and closer to the values of DTT, especially for the $\lambda 8542$ and the $\lambda 8662$ lines. The differences are not that small in the case of the $\lambda 8498$ line. Although the choice of line windows does affect the measured EQWs and largely accounts for the differences in the $\lambda \lambda 8542,8662$ lines, it must be noted that the reason for part of the discrepancy in the EQW values (especially those of the $\lambda 8498$ ) lies in the choice of the continuum.

\section{Ca II triplet strengths as a function of} temperature, surface gravity \& metallicity

\subsection{Temperature}

Figures 3a-d show the $\lambda \lambda 8498,8542$ and the $\lambda 8662$ spectra, each figure for a given luminosity and roughly the same metallicity (within $\Delta[\mathrm{Fe} / \mathrm{H}]= \pm 0.25$ ) spread over the range of spectral types from F8 to M3. These figures are for luminosity types IV, II, Ib and 0-Ia. Each set of spectra for a given luminosity hardly shows any variation with spectral type. It is true for all luminosities and for both metal rich and metal poor stars. Figure 4 gives the plot between the Ca II EQW (CaT) and $(R-I)$. The filled symbols correspond to stars with $[\mathrm{Fe} / \mathrm{H}] \geq 0.0$ whereas the unfilled ones to stars with $[\mathrm{Fe} / \mathrm{H}]<0.0$. This is true for all the subsequent figures. CaT represents the sum of the EQWs of all the $3 \mathrm{Ca}$ II triplet lines. The EQWs of all the 3 lines are added to improve the $\mathrm{S} / \mathrm{N}$ ratio and also to eliminate the influence of irrelevant lines. Although the dominant effect is that of luminosity, the plot essentially looks like a scatter diagram, especially when one looks at the distribution of dwarfs, giants and supergiants separately. There is a random spread of EQW over $(R-I)$, particularly so in the case of giants and supergiants. There appears to be a conglomerat of stars in the lower left part of the diagram. It could be a selection effect, for the program list has very few $\mathrm{M}$ stars and does not extend to large enough $(R-I)$. The theoretical calculations of ErdelyiMendes \& Barbuy (1991), however, show that the Ca II EQWs are sensitive to $T_{\text {eff. }}$ Zhou (1991) in his observational study of a large number of stars has detected a trend of EQWs decreasing with increase in $(R-I)$ but only in the domain containing $\mathrm{M}$ stars $((R-I) \geq 1.4)$. In our sample also, for example, Fig. 3d shows a similar effect. The CaT is weaker in $\mu$ Cep (M2 Iae). It could be because of less Ca II at lower temperatures. However, it could also be an observational artifact because of the $\mathrm{TiO}$ bands getting stronger for cooler stars, lowering the continuum level and the EQWs. DTT (1989) found no strong relationship between the Ca II strengths and the temperature. For the sample of stars we have, we also infer that CaT hardly reveals any dependence on effective temperature.

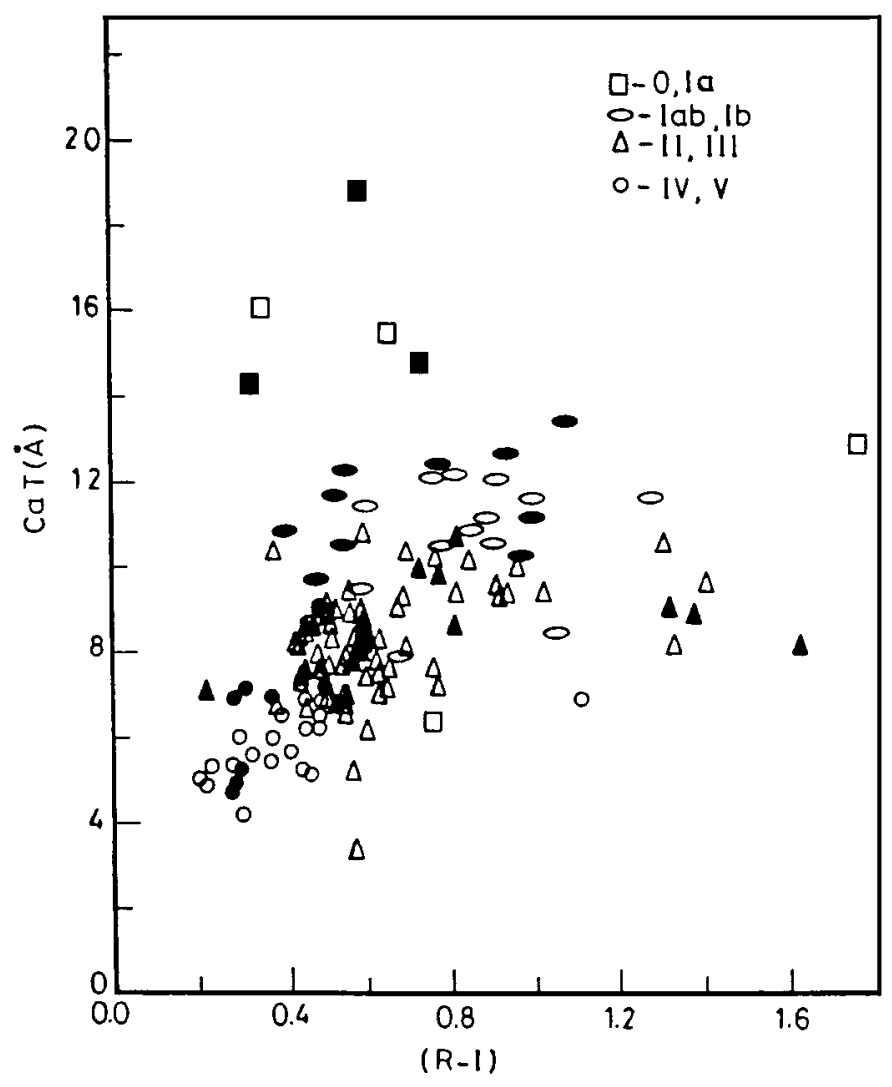

Fig. 4. CaT (sum of the EQWs of the 3 lines) versus $(R-I)$. $\square, \bigcirc, \triangle$, ○ represent superluminous supergiants, supergiants, bright giants + giants, subgiants + dwarfs respectively. The filled symbols belong to stars with $[\mathrm{Fe} / \mathrm{H}] \geq 0.0$ whereas the unfilled ones to those with $[\mathrm{Fe} / \mathrm{H}]<0.0$. The same symbols hold for the subsequent figures

The detailed non-LTE calculations of the Ca II triplet equivalent widths carried out by JCJ over a broad range of input parameters, i.e., $\log g$ between 0.0 and $4.0, T_{\text {eff }}$ from $4000 \mathrm{~K}$ to $6600 \mathrm{~K}$ and $\mathrm{Ca}$ abundance between +0.2 and -1.0 show that calcium is predominantly singly ionized in the line forming region in the entire parameter space covered. This explains the lack of sensitivity of Ca II EQW to temperature for stars under consideration. For cooler stars however, there is more and more of neutral calcium; Ca II is no longer the dominant ionization species and that explains the decrease of Ca II EQW with temperature. JCJ's computations show that the response of the Ca II EQW to $T_{\text {eff }}$ is different for different values of $\log g$. For 

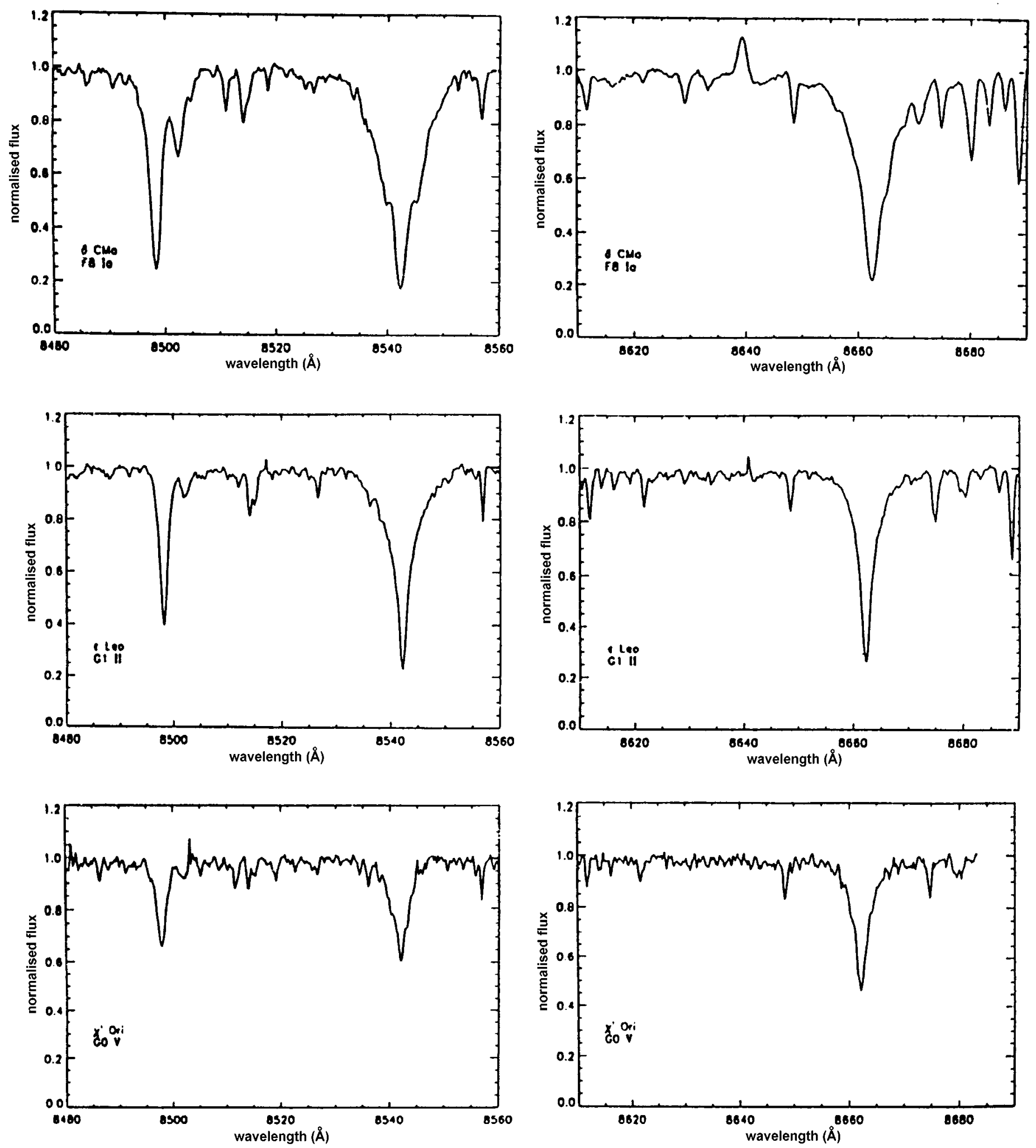

Fig. 5. Sample normalised spectra around Ca II $\lambda \lambda 8498,8542,8662$ for stars of each spectral type over a range of luminosities: a) F8-G1, b) G8-G9, c) K2-K4 
(b)
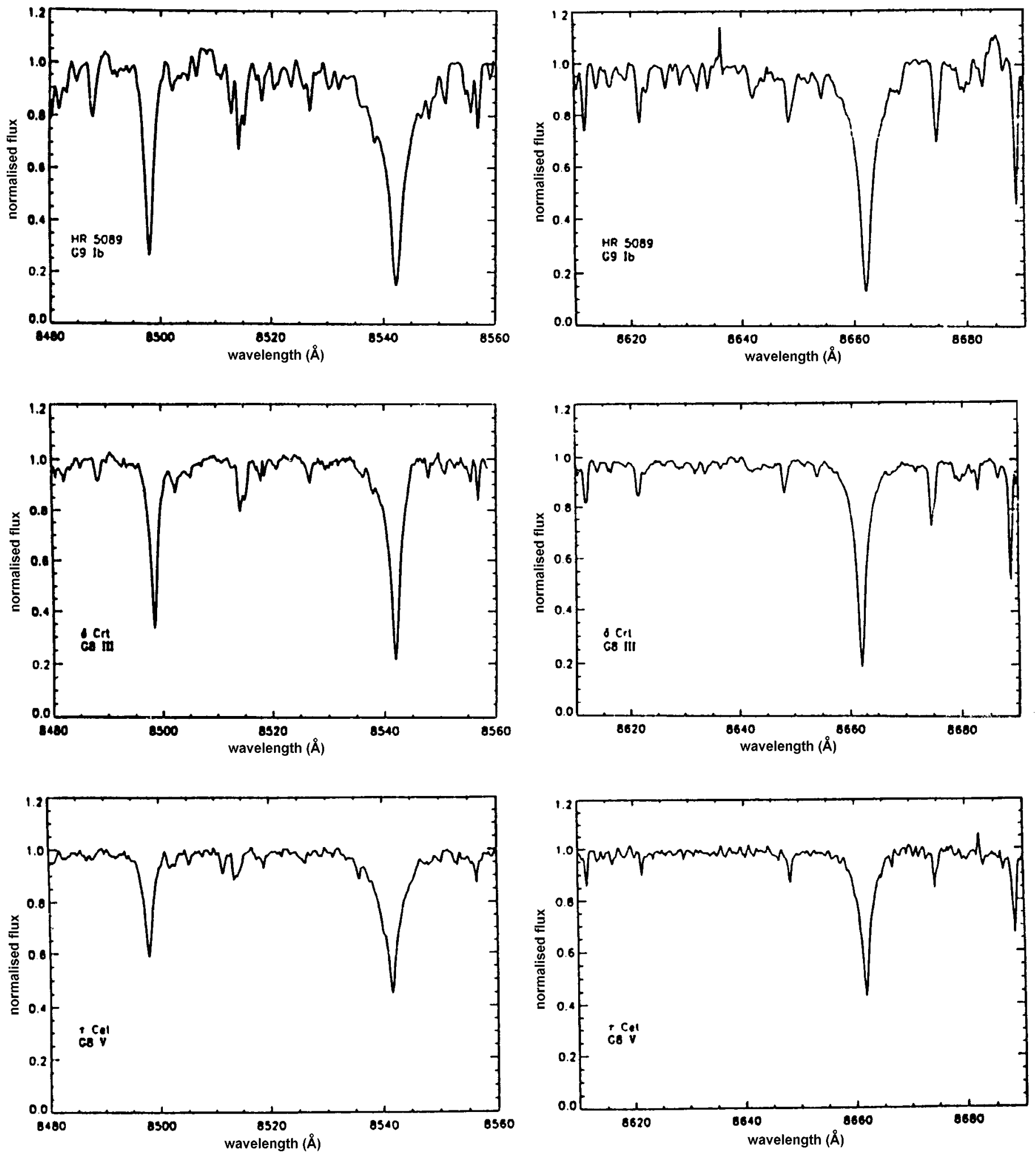

Fig. 5. continued 
(c)
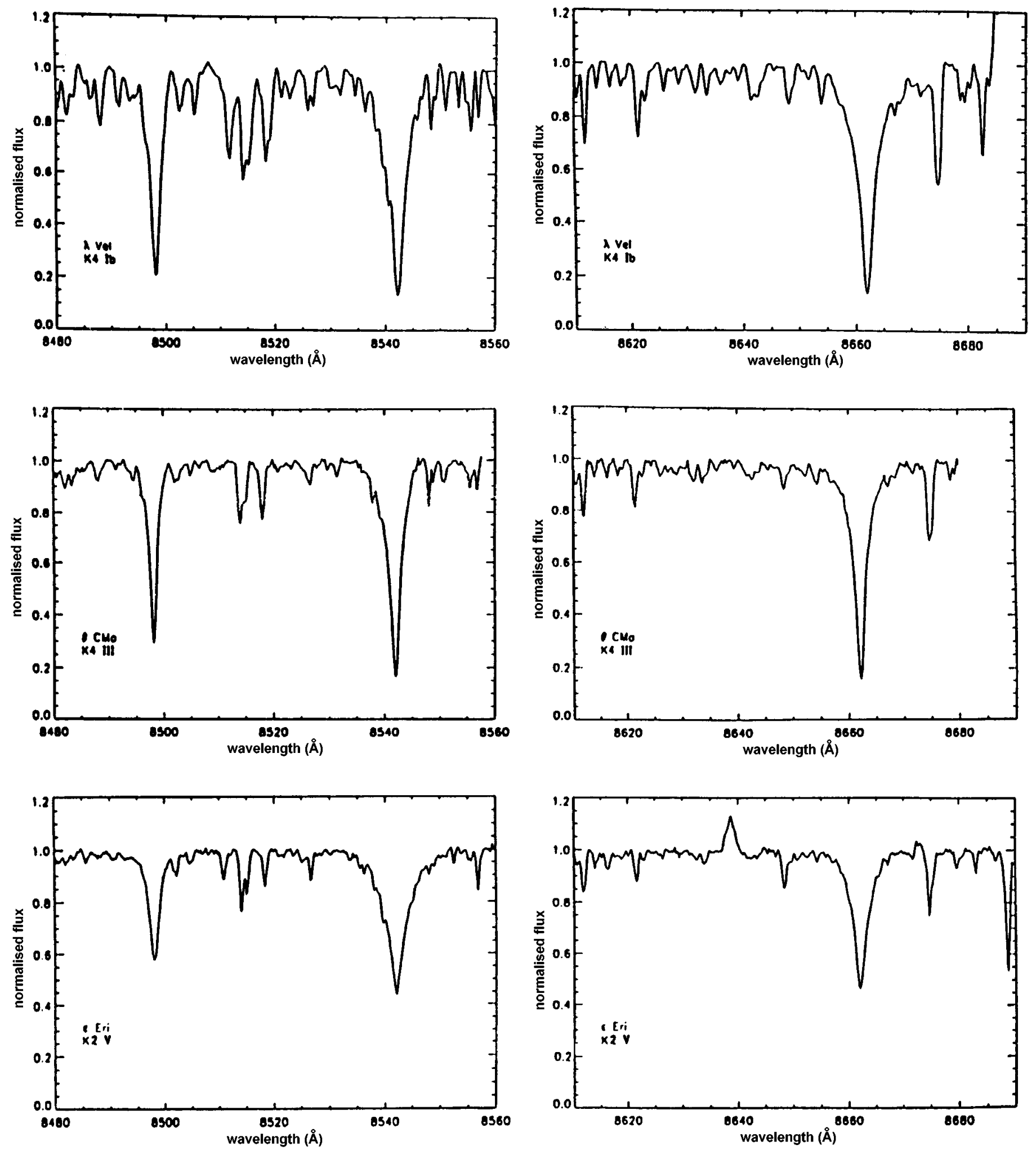

Fig. 5. continued 
large values of $g$, the EQW decreases monotonically with temperature; for lower $\log g$, there is a clear convex shape. The present observations display a similar behaviour there is a trend of EQW increasing when $T_{\text {eff }}$ decreases for dwarfs and there is a mild suggestion of convex appearance in the case of giants and supergiants. JCJ emphasize that, if models of only a limited temperature range are available, one would conclude on a single-valued dependence of EQW on gravity, independent of temperature. Figure 4 also shows the importance of considering a large span in temperature; without this only the gravity effect will show up.

\subsection{Surface gravity}

In Figs. 5a-c are displayed representative spectra in $\lambda \lambda 8498,8542$ and $\lambda 8662$ for stars over the entire range of luminosities, of roughly the same spectral type and metallicity (within $\Delta[\mathrm{Fe} / \mathrm{H}]= \pm 0.2$ ). The 3 different sets of spectra a to c are for 3 different spectral types, namely, F8-G0, G8-G9 and K2-K4. These spectra clearly reveal a strong correlation between the Ca II triplet strengths and the luminosity. The lines are strong, wide and deep in supergiants and much weaker in dwarfs. Such a strong dependence on luminosity holds true for all spectral types, independent of $[\mathrm{Fe} / \mathrm{H}]$. One would, however, notice that the dependence of $\mathrm{CaT}$ on luminosity is not as striking in metal poor stars (see Fig. 5b) as in the stars of solar metallicity (Fig. 5a) or in more metal rich stars (as shown in Fig. 5c). Figure 5a also includes a superluminous supergiant $\delta$ CMa which shows unusually strong and wide asymmetric Ca II triplet absorption features combined with emission, which suggests a complex velocity structure in the atmosphere of such stars.

Figure 6a shows the plot of CaT against $\log g$ for the whole sample. The plot includes eight hypergiants marked by squares and many more dwarfs and subgiants. More importantly, the data now include many more metal poor stars and stars over a much larger range of $[\mathrm{Fe} / \mathrm{H}]$. The $\mathrm{Ca}$ II EQWs obviously anticorrelate very strongly with $\log g$. This is analytically well understood that the ionized calcium lines (being the most abundant ionization species in the domain considered here) strengthen in response to an increase in electron density for stars where $\mathrm{H}^{-}$dominates the continuous opacity. The increase in the EQW is not due to an increase in the line absorption coefficient; instead it is due to a decrease in the continuous absorption coefficient. The relationship is not linear. The EQW decreases rapidly with increasing $\log g$ for supergiants and giants; whereas the relation is flatter for subgiants and dwarfs. In other words, there is a very strong increase in EQW (from $8.0 \AA$ to $16.0 \AA$ ) for small $\log g$ 's (from 2.0 to 0.0 ) and a milder increase from $4.0 \AA$ to $8.0 \AA$ for higher $\log g$ 's (from 4.5 to 2.0). The strong increase in EQW for small $\log g$ values compared to the modest increase for higher $\log g$ values agrees very well with the theoretical computations of JCJ. JCJ have calculated the equivalent width $W$ (sum of EQWs of $\lambda 8542$ and $\lambda 8662$ ) as a function of $\log g$ for several metallicities and have in each case found that the relationship between $W$ and $\log g$ represents a least squares, second order fit in the variable $g$ over the large parameter space considered by them. The observational data in the past have not led to a second order term in the fit because of the sparse observations for high luminosities. As emphasised by JCJ, it is important to represent the high luminosity stars well for fitting to integrated spectra of external galaxies since they often contribute a large fraction of the integrated light. A linear fit to EQW obtained from the most common nearby stars, i.e., dwarfs, if used to calibrate the integrated spectrum of an external galaxy would lead to an underestimate of the ratio of dwarfs to giants in the galaxy. The present observations however, include other than those of a large number of Ib supergiants, also of 8 superluminous supergiants (0-Ia) denoted by squares in Fig. 6 . Just these many are adequate to give a steep relationship for low $\log g$ 's and a relationship over the entire parameter space similar to the second order fit of JCJ. It has thus been crucial to add the observational data of the luminous supergiants. The dark smooth curve drawn on each of the Figs. 6a, $\mathrm{b}$ and $\mathrm{c}$ is a second order polynomial fit to the data points. Data points that deviate by large amounts from the correlation pattern were excluded in obtaining these fits. Figure 6 a shows that the luminous supergiants perhaps require a steeper fit. The theoretical computations of Erdelyi-Mendes \& Barbuy (1991) also predict a non-linear response. JCJ have tabulated non-LTE values of $W$ for $\log g=0.0,1.0,2.0,3.0,4.0$ and $T_{\text {eff }}=4000 \mathrm{~K}, 5000 \mathrm{~K}$, $6000 \mathrm{~K}, 6600 \mathrm{~K}$ and metallicities of $+0.2,0.0,-0.5$ and -1.0 . We have compared the observed values of $W$ given in Table 2 of all our program stars with the theoretical $W$ for the closest possible match of the parameters and find that the two measures match rather very well. The theoretical $W$ tends to be slightly higher than the observational values probably because JCJ in their calculations of the EQWs of $\lambda 8542$ and $\lambda 8662$ chose the boundaries in wavelength for the wings as defined in the work of DTT (from $\lambda 8527$ to $\lambda 8557$ and $\lambda 8647$ to $\lambda 8667$ respectively) to enable direct comparison with DDT's observations. The boundaries chosen in our EQW measurements are smaller as has been elucidated earlier.

The large scatter in the plot is partly due to the varying distribution of metal content in stars. JAJ have shown that the deviations of the observed points from the above relationship are related to metallicity. This effect is seen in the Fig. $6 \mathrm{a}$ for stars marked by their $[\mathrm{Fe} / \mathrm{H}]$. In Figs. 6b and c, CaT has been plotted against the subsamples of metal rich stars (defined by $[\mathrm{Fe} / \mathrm{H}] \geq 0.0$ ) and of metal poor stars $([\mathrm{Fe} / \mathrm{H}]<0.0)$ respectively. Evidently, a much tighter relation between $\mathrm{CaT}$ and $\log g$ exists for metal rich stars as found in the observational study by JAJ and DTT. The theoretical calculations of JCJ also show that the 

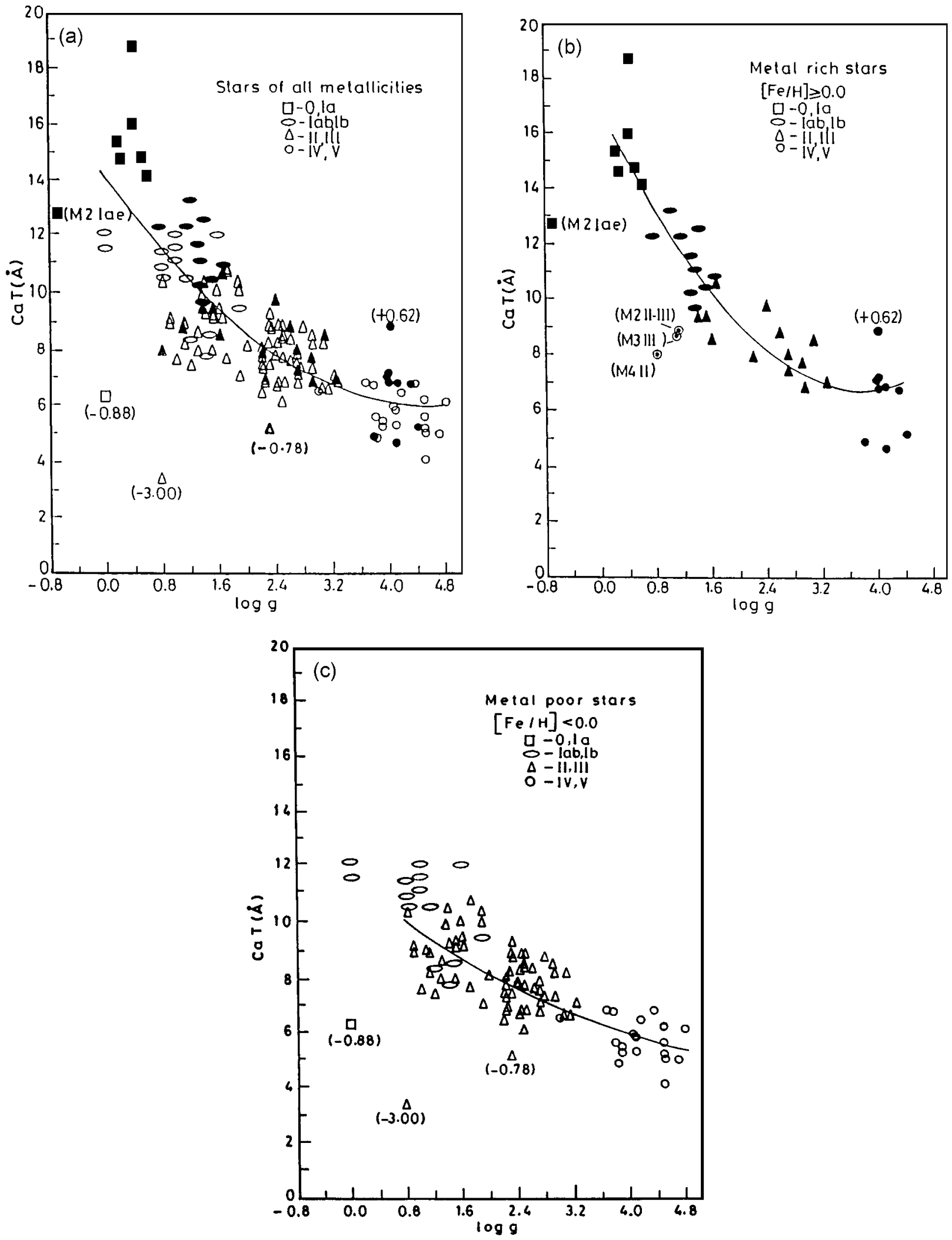

Fig. 6. CaT versus $\log g$ : a) for the whole star sample, b) for metal rich stars $([\mathrm{Fe} / \mathrm{H}] \geq 0.0)$, c) for metal poor stars $([\mathrm{Fe} / \mathrm{H}]<0.0)$ The smooth curve drawn is a second order polynomial fit to the data points 
relative dependence of EQW on $\log g$ is highest for high metallicity systems. For high metallicity the change in EQW inside their computed range of $T_{\text {eff }}$ is much smaller than the changes obtained by varying $\log g$ from 4 to 0 . This makes the EQW- $\log g$ relation a useful one in the study of integrated spectra and population synthesis of high-metallicity stellar systems.

$[\mathrm{Fe} / \mathrm{H}]$ is known for only 4 out of the 8 hypergiants observed. 3 of them have $[\mathrm{Fe} / \mathrm{H}]>0.0$ and the other one is metal poor. Being superluminous and hence massive, most of them must be young. Therefore, we have assumed $[\mathrm{Fe} / \mathrm{H}] \geq 0.0$ for the remaining 4 hypergiants and plotted them accordingly on the plot for the sample of metal rich stars (Fig. 6b). 4 out of the 5 stars that show large deviation from the strong correlation are all $\mathrm{M}$ stars as marked on the figure. Because of the strong $\mathrm{TiO}$ bands appearing in their spectrum and hence uncertainties in the continuum location, their EQWs are suspected to have been underestimated. Leaving these aside, the data for the metal rich stars clearly display a very steep non-linear response. The metal poor stars as seen in Fig. 6c, do not follow such a close relationship; it is non-linear although is not as steep. The stars that deviate by large amounts are especially metal poor. DTT found a very poor dependence of the Ca II EQW on $\log g$ for metal poor stars and suggested that since the Ca II lines are easily measurable even in stars with very low metal content, they could be used as a metallicity indicator for metal poor stellar systems. JCJ's computations also give a similar picture for stars of lower metallicity. On the other hand, the close-knit relationship between the CaT strengths and $\log g$ for metal rich stars could be used to determine the luminosity of stellar systems of metallicity above solar values from the observed Ca II triplet strengths once a good calibration is established.

\subsection{Metallicity}

Figures 7a-c demonstrate for $\lambda \lambda 8498,8542$ and $\lambda 8662$ how their strengths relate to $[\mathrm{Fe} / \mathrm{H}]$. As stated earlier, $[\mathrm{Fe} / \mathrm{H}]$ directly reflects the abundance of heavy elements including calcium. Under this assumption, CaT can be considered as a reliable measure of metallicity. The spectral type and $[\mathrm{Fe} / \mathrm{H}]$ are specified for each star on its spectrum. Each set of spectra describes a range of metallicity as large as possible for a given luminosity and a given spectral type (or within a very small range of that spectral type). Each such set of spectra shows the variation of the Ca II triplet strengths with $[\mathrm{Fe} / \mathrm{H}]$ in the sense that the Ca II triplet lines are much stronger in metal rich stars than in metal poor ones. Dwarfs are most likely to cover a larger range in $[\mathrm{Fe} / \mathrm{H}]$ because of the spread in age that exists among them; hence it is possible to study the metallicity effects better in dwarfs. Figure 7a does cover a wide range in $[\mathrm{Fe} / \mathrm{H}]$. Supergiants, being young Population I objects, are mostly massive stars born out of the richer interstellar medium and therefore are not expected to cover a variation in metallicity as large as that covered by the dwarf sample. Figure $7 \mathrm{c}$ however, includes stars spanning a large enough range in $[\mathrm{Fe} / \mathrm{H}]$. Also, Fig. 7b for giants includes HR 5270 which has an extremely low EQW in all the 3 lines, because its $[\mathrm{Fe} / \mathrm{H}]$ is as low as -3.0 . In general, however, a close look at Fig. 7 reveals that although the dwarfs cover a larger range in $[\mathrm{Fe} / \mathrm{H}]$ than the supergiants, the variation in the Ca II strengths is more prominent among the supergiants. This will be more obvious in the plots in Figs. 8a, b, c and d. It is also apparent in Fig. 7 that the variation with respect to metallicity is milder than with respect to luminosity.

A plot between $\mathrm{CaT}$ and $[\mathrm{Fe} / \mathrm{H}]$ is shown in Fig. 8a for the whole sample of stars with known $[\mathrm{Fe} / \mathrm{H}]$. A large part of the scatter is due to the luminosity effect; dwarfs are way down, separated from giants higher up and supergiants still higher. If the star with $[\mathrm{Fe} / \mathrm{H}]=-3.0$ is included, the spread in metallicity is over a factor of 10000 ; otherwise it is still over a factor of 400 . The response of CaT to metallicity is not linear over this metallicity range. Instead, CaT increases exponentially with $[\mathrm{Fe} / \mathrm{H}]$. This further corroborates that it is essential to have as wide a parameter space as possible. Theoretical calculations of JCJ also predict a non-linear response - a complex behaviour where the dependence of $W$ on metallicity is a strong function of $\log g$. Figures $8 \mathrm{~b}, \mathrm{c}$ and d clearly demonstrate an exactly similar behaviour. The $\mathrm{CaT}-[\mathrm{Fe} / \mathrm{H}]$ correlation is much stronger for the supergiants than for the dwarfs. In the case of supergiants, the dependence is sharp and steep, even over a limited range of $[\mathrm{Fe} / \mathrm{H}]$. The EQWs differ by a factor of 2.5 over metallicity range from -0.9 to +0.2 , i.e., a factor of 12 . The response is milder in the case of giants and dwarfs. Over the same $[\mathrm{Fe} / \mathrm{H}]$ range, EQWs differ by a factor of 1.5 in giants and even less in dwarfs. A factor of 2.5 only in EQW for giants occurs over a metallicity factor as large as 1000 . The metallicity dependence is much stronger for supergiants although the covered range in metallicity is smaller.

\section{Chromospheric activity}

The 8 hypergiants that have been observed show that the Ca II line profiles have a complex structure. Emission components appear within the absorption profile, perhaps similar to the ones that are observed in the $\mathrm{Ca}$ II $\mathrm{H}$ and $\mathrm{K}$ profiles of stars known to have chromospheric activity. However, the rest of the stars, i.e., the dwarfs, giants and supergiants show only absorption profiles in the Ca II triplet lines. Anderson (1974) observed the $\lambda 8498$ line in 28 stars of spectral types F8-M2 at a resolution of $0.28 \AA$ and found no distinct emission in any star. Only one star, $\beta$ Dra showed a central residual intensity (CRI) larger than in the other stars, possibly due to filling in by chromospheric emission. This star is the only one with a very active chromosphere in Anderson's list (intensity 

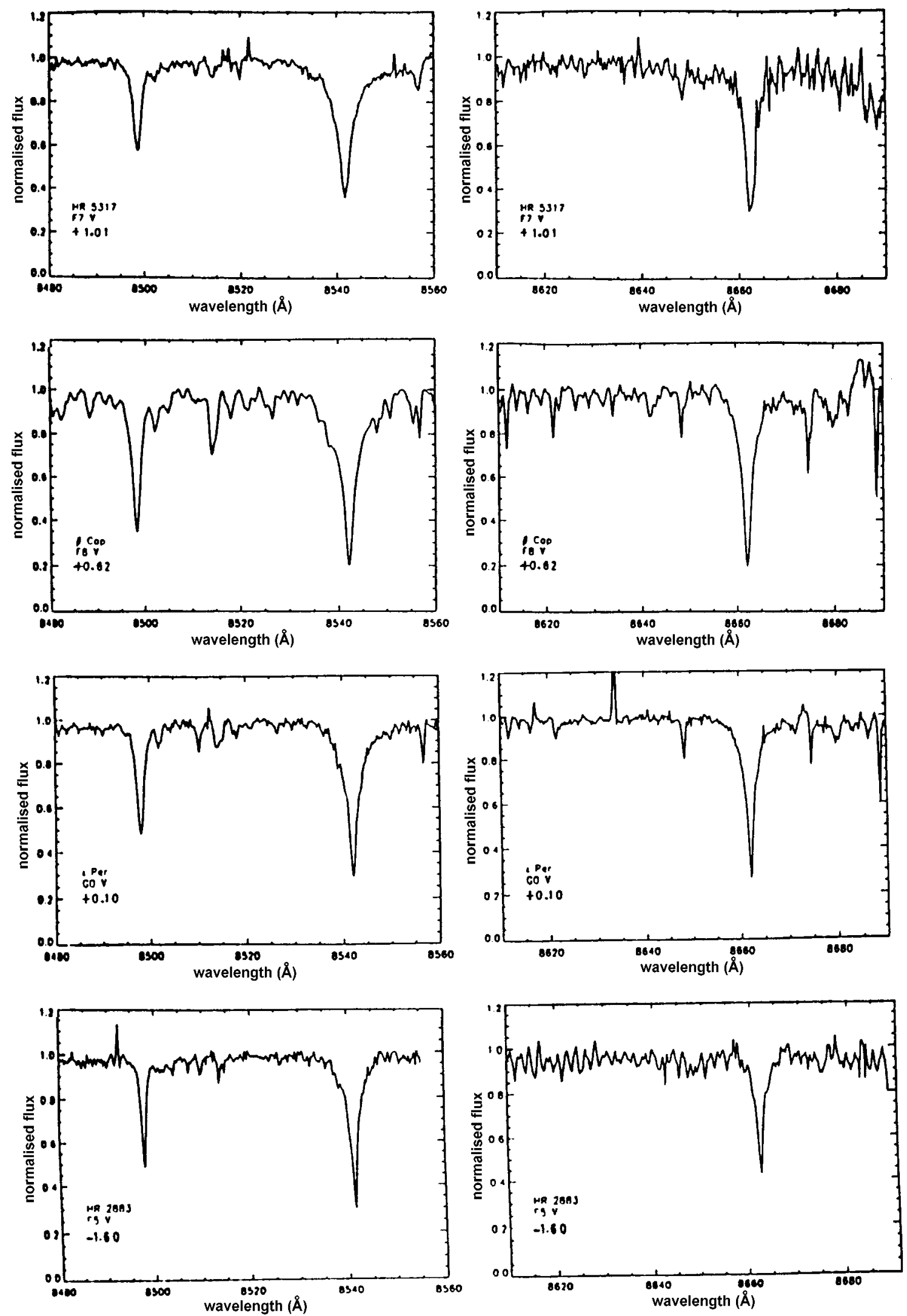

Fig. 7. Representative normalised spectra around the $\lambda \lambda 8498,8542,8662$ lines for stars of a given luminosity and spectral type (or within a very small range of that spectral type) over a range of metallicity: a) V, F5-G0; b) II, K3; c) 0-Ia, F8-G4. Spectrum of each star is denoted by the spectral type and $[\mathrm{Fe} / \mathrm{H}]$ 
(b)
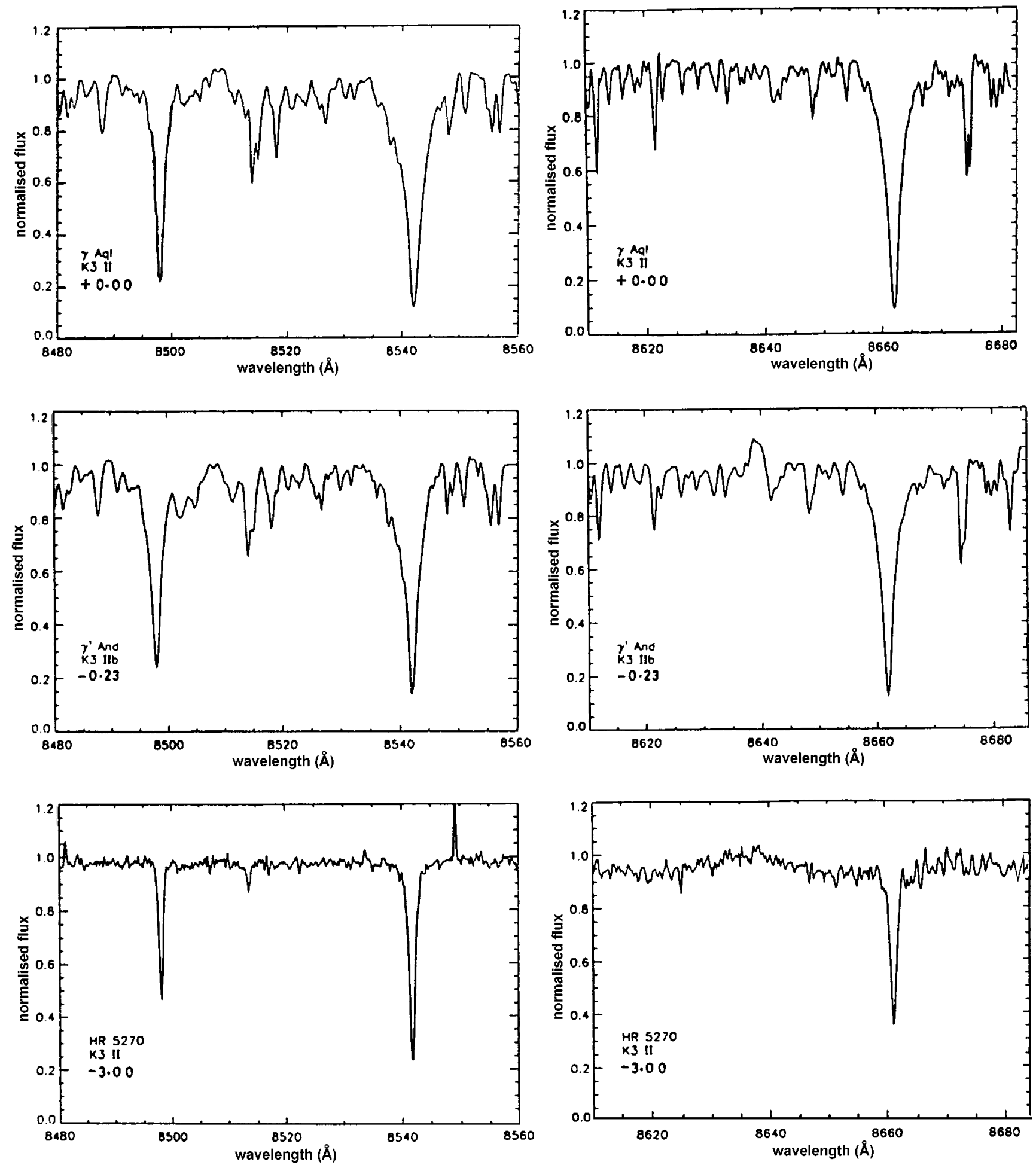

Fig. 7. continued 
(c)
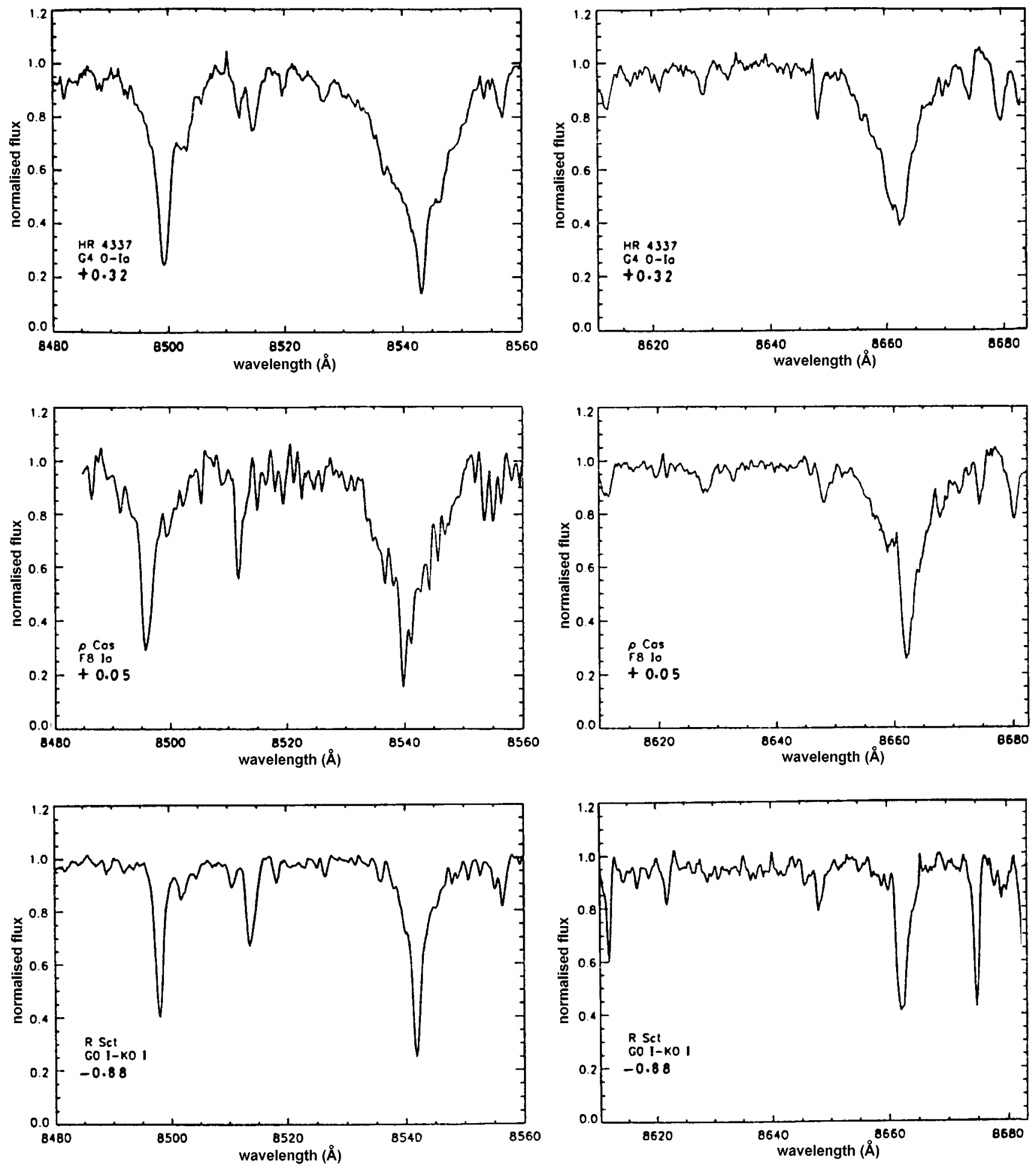

Fig. 7. continued 
Table 3. Summary of observations of star pairs

\begin{tabular}{|c|c|c|c|c|c|c|c|c|c|}
\hline \multirow[t]{2}{*}{$\overline{\text { Star }}$} & \multirow{2}{*}{$\begin{array}{l}\text { Spectral } \\
\text { type }\end{array}$} & \multirow[t]{2}{*}{$I(\mathrm{CaII} \mathrm{K})$} & \multirow{2}{*}{$\begin{array}{l}v \sin i \\
\left(\mathrm{~km} \mathrm{~s}^{-1}\right)\end{array}$} & \multicolumn{3}{|c|}{ CRI } & \multicolumn{3}{|c|}{ EQW } \\
\hline & & & & $\lambda 8498$ & $\lambda 8542$ & $\lambda 8662$ & $\lambda 8498$ & $\lambda 8542$ & $\lambda 8662$ \\
\hline$\overline{\mathrm{HR}} 2269$ & K3 Ib & - & - & 0.32 & 0.25 & 0.30 & 1.59 & 3.40 & 3.36 \\
\hline 3 Cet & K3 Ib & - & $<17$ & 0.18 & 0.12 & 0.20 & 2.11 & 4.66 & 3.63 \\
\hline HR 2269 & K3 Ib & - & - & 0.32 & 0.25 & 0.30 & 159 & 3.40 & 3.36 \\
\hline$\eta$ Per & K3 Ib & 4 & $\leq 54$ & 0.17 & 0.12 & 0.08 & 2.52 & 4.59 & 3.96 \\
\hline $56 \mathrm{Peg}$ & G8 Ib & 4 & $<17$ & 0.45 & 0.35 & 0.24 & 1.50 & 3.43 & 2.85 \\
\hline$\zeta$ Cep & $\mathrm{K} 1.5 \mathrm{Ib}$ & 3 & $<17$ & 0.16 & 0.11 & 0.10 & 2.60 & 5.17 & 4.51 \\
\hline $56 \mathrm{Peg}$ & G8 Ib & 4 & $<17$ & 0.45 & 0.35 & 0.24 & 1.50 & 3.43 & 2.85 \\
\hline$\epsilon \mathrm{Gem}$ & G8 Ib & 4 & $<17$ & 0.19 & 0.15 & 0.11 & 2.62 & 4.71 & 4.02 \\
\hline$\beta$ Dra & G2 Ib-IIa & 3 & 13 & 0.37 & 0.27 & 0.31 & 1.83 & 4.40 & 3.43 \\
\hline$\zeta \mathrm{Gem}$ & G0 Ib & - & $\leq 54$ & 0.28 & 0.15 & 0.15 & 1.76 & 4.66 & 4.35 \\
\hline$\delta$ Vir & M3 III & 4 & - & 0.33 & 0.20 & 0.17 & 1.59 & 3.37 & 3.05 \\
\hline$\eta \mathrm{Gem}$ & M3 III & 5 & - & 0.28 & 0.14 & 0.18 & 1.85 & 3.89 & 3.36 \\
\hline$\theta \mathrm{CMa}$ & K4 III & 3 & $<19$ & 0.30 & 0.17 & 0.16 & 1.49 & 2.94 & 2.62 \\
\hline$\alpha \mathrm{Tau}$ & K5 III & 3 & $<17$ & 0.25 & 0.12 & 0.13 & 1.88 & 4.20 & 3.22 \\
\hline$\nu$ Hya & K2 III & 3 & $<17$ & 0.33 & 0.23 & 0.18 & 1.32 & 3.01 & 2.84 \\
\hline$\beta \mathrm{Col}$ & K2 III & 2 & - & 0.28 & 0.15 & 0.17 & 1.57 & 3.46 & 2.94 \\
\hline$\sigma \mathrm{Gem}$ & K1 III & - & 22 & 0.65 & 0.51 & 0.56 & 1.31 & 3.23 & 2.09 \\
\hline$\beta \mathrm{Gem}$ & K0 IIIb & 1 & $<17$ & 0.37 & 0.17 & 0.23 & 1.37 & 3.53 & 2.64 \\
\hline$\lambda$ And & G8 III & 5 & $<19$ & 0.73 & 0.57 & 0.58 & 0.86 & 2.58 & 1.71 \\
\hline$\phi^{2}$ Ori & K0 III & 2 & - & 0.37 & 0.20 & 0.23 & 1.20 & 2.85 & 2.52 \\
\hline$\epsilon$ Hya & G5 III & - & 19 & 0.5 & 0.33 & 0.36 & 1.15 & 3.04 & 2.35 \\
\hline o UMa & G5 III & 1 & 17 & 0.38 & 0.21 & 0.12 & 1.30 & 3.27 & 2.21 \\
\hline$\phi$ Vir & G2 IV & 3 & 0 & 0.58 & 0.50 & 0.43 & 1.06 & 2.36 & 2.01 \\
\hline$\mu$ Her & G5 IV & 0 & 20 & 0.45 & 0.22 & 0.29 & 1.10 & 3.48 & 2.28 \\
\hline$\phi$ Vir & G2 IV & 3 & 0 & 0.58 & 0.50 & 0.43 & 1.06 & 2.36 & 2.01 \\
\hline$\zeta$ Her & G0 IV & 0 & $\leq 10$ & 0.47 & 0.33 & 0.25 & 1.18 & 2.28 & 2.07 \\
\hline$\epsilon$ Eri & $\mathrm{K} 2 \mathrm{~V}$ & 4 & $<17$ & 0.58 & 0.44 & 0.47 & 1.16 & 2.96 & 2.06 \\
\hline$\phi^{2}$ Eri & $\mathrm{K} 1 \mathrm{~V}$ & 2 & - & 0.50 & 0.32 & 0.33 & 1.32 & 3.11 & 2.38 \\
\hline$\tau$ Cet & G8 V & - & 2 & 0.54 & 0.40 & 0.43 & 0.97 & 2.31 & 1.80 \\
\hline 82 Eri & G8 V & - & - & 0.48 & 0.31 & 0.33 & 1.07 & 3.04 & 2.37 \\
\hline$\chi^{1}$ Ori & G0 V & 2 & 6 & 0.66 & 0.60 & 0.46 & 0.90 & 1.66 & 1.58 \\
\hline$\beta \mathrm{Com}$ & G0 V & 1 & 6 & 0.57 & 0.37 & 0.37 & 0.90 & 2.29 & 2.02 \\
\hline$\chi^{1}$ Ori & G0 V & 2 & 6 & 0.66 & 0.60 & 0.46 & 0.90 & 1.66 & 1.58 \\
\hline$\beta$ Vir & F9 V & 0 & 3 & 0.50 & 0.35 & 0.33 & 1.10 & 1.88 & 1.83 \\
\hline HR 5317 & F7 V & - & 30 & 0.58 & 0.37 & 0.32 & 1.17 & 3.40 & 2.17 \\
\hline$\beta$ Cap & F8 V & - & 54 & 0.36 & 0.21 & 0.21 & 1.78 & 4.27 & 2.84 \\
\hline
\end{tabular}



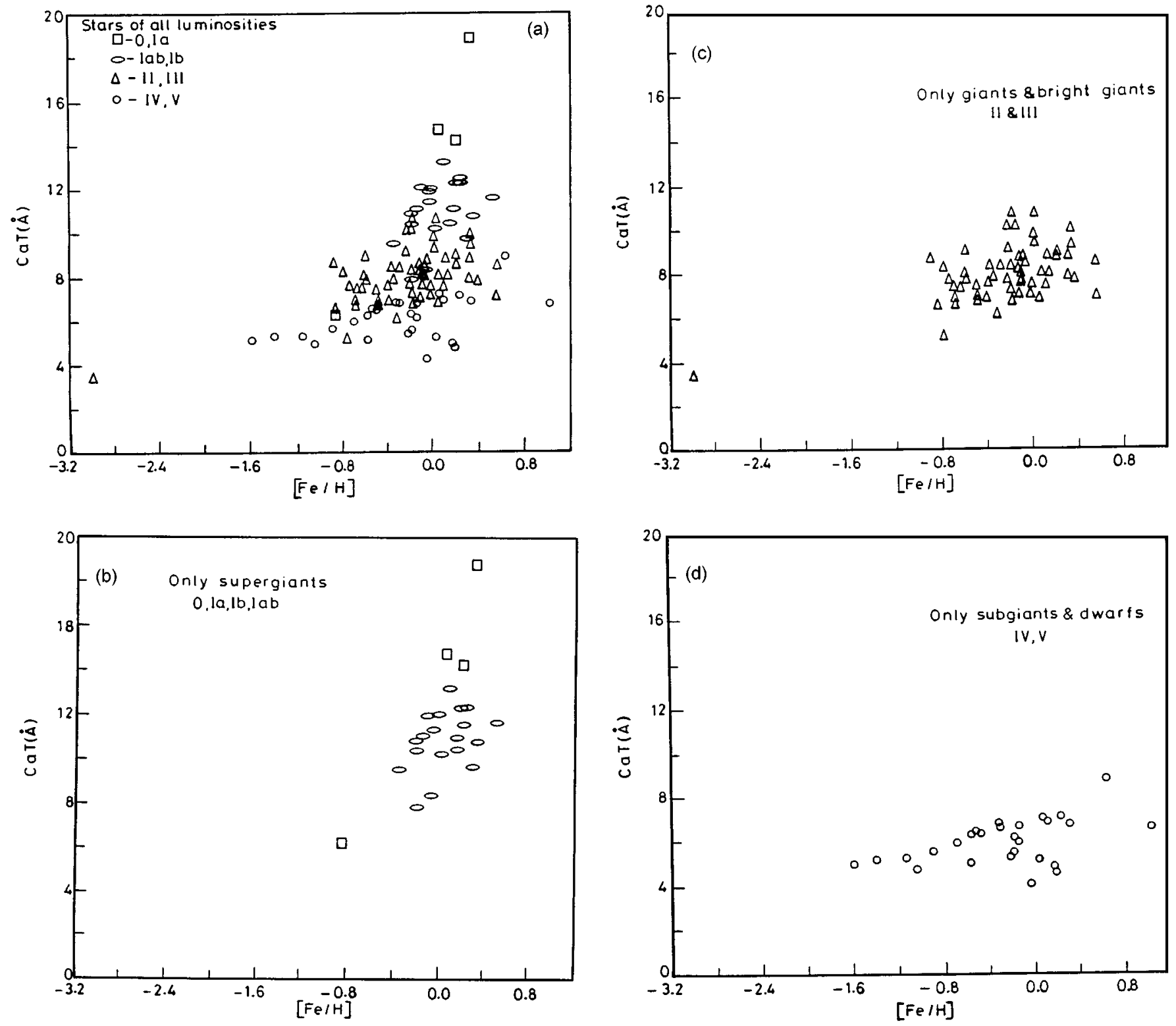

Fig. 8. CaT versus $[\mathrm{Fe} / \mathrm{H}]$ for: a) the whole sample, b) supergiants, c) giants, d) dwarfs

class 3 according to Wilson 1976). Linsky et al. (1979) also observed $\lambda 8542$ profiles at a resolution of $0.14 \AA$ in 49 stars as a probe for chromospheric activity. Their resolution is twice as high as that of Anderson and is sufficient to resolve emission features within the absorption profiles of the Ca II triplet in stars if they are present. No evidence of emission was found even for the most active chromosphere stars.

However, it was found that when the observed spectra of stars are arranged into groups with similar spectral type and luminosity class, within each group there are stars for which the line profiles differ in shape near the line center from the rest of the stars in that group, in the sense that CRI is higher for these stars compared to that of the rest. Some of the examples cited by Linsky et al. (1979) are 111 Tau- $\beta$ Vir, $\xi$ Boo A- $\tau$ Cet, $\lambda$ And- $\delta$ Crt, $\chi^{1}$ Ori- $\beta$ Com, $\sigma$ Gem- $\beta$ Cet, $\alpha$ Aur- $\beta$ Crv, $\beta$ Dra- $\epsilon$ Leo, 9 Peg- $\epsilon$ Gem; the first one in each pair having a higher CRI than the other. These are perhaps the stars whose absorption profiles show filling in of the $\lambda 8542$ cores by unresolved chromospheric emission. According to Wilson (1976), most of these are the active chromosphere stars with an intensity index higher than 3. Following Linsky et al. (1979), we have also grouped all the observed stars of similar spectral type, luminosity and also metallicity (within $\Delta[\mathrm{Fe} / \mathrm{H}]= \pm 0.2$ ) and have found about 13 pairs that are tabulated in Table 3 . The first one is with the larger CRI than the other and is therefore suspected to 

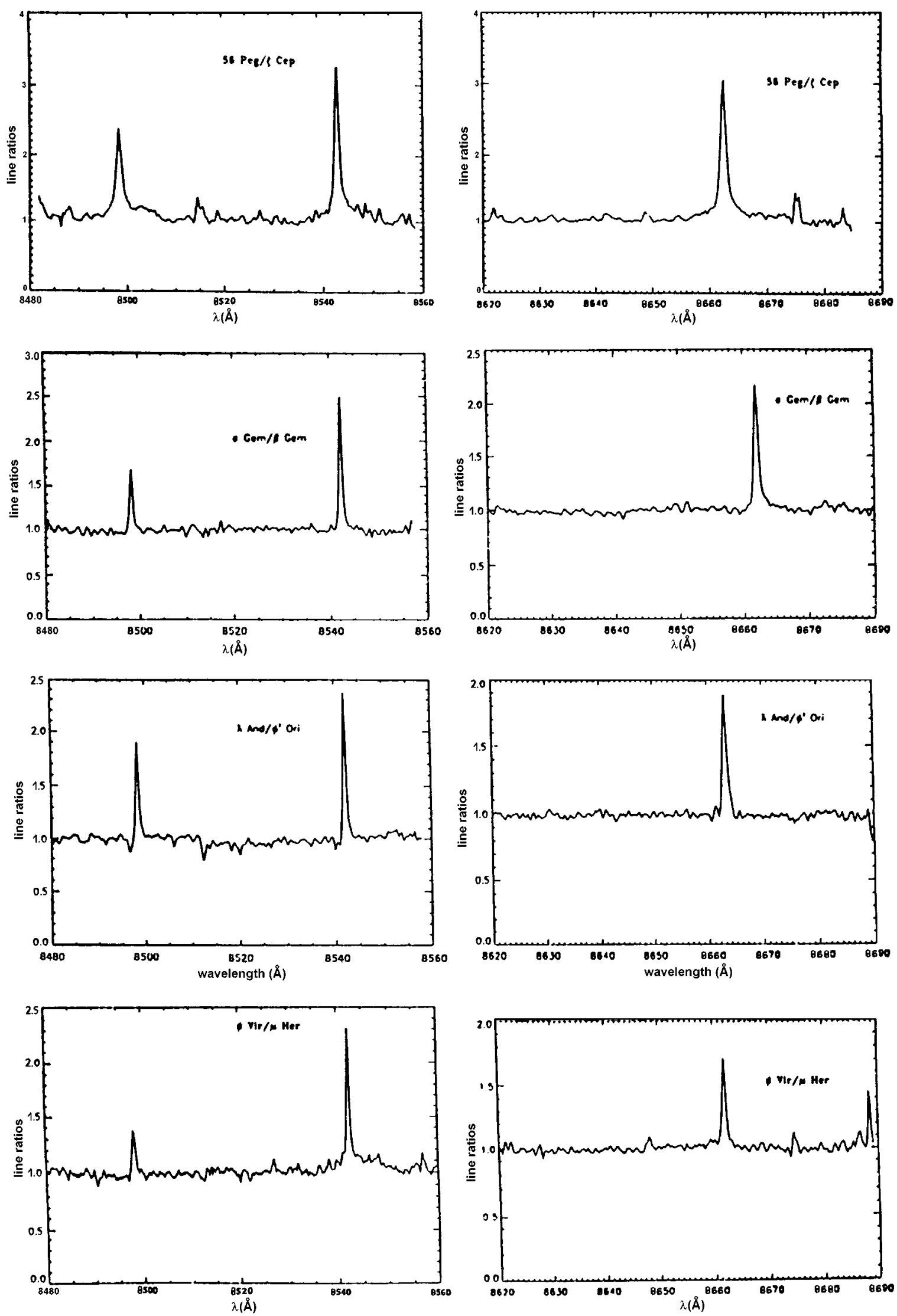

Fig. 9. Line ratio spectra of $\lambda \lambda 8498,8542,8662$ of a few sample pairs 
Table 4. Emission parameters of ratio spectra

\begin{tabular}{|c|c|c|c|c|c|c|c|c|c|c|}
\hline \multirow[t]{2}{*}{$\overline{\text { Ratio }}$} & \multirow[t]{2}{*}{ spectra } & \multicolumn{3}{|c|}{$\mathrm{FWHM}(\AA)$} & \multicolumn{3}{|c|}{$\begin{array}{l}\text { emission } \\
\operatorname{peak}(\AA)\end{array}$} & \multicolumn{3}{|c|}{$\begin{array}{l}\text { area of } \\
\text { emission }\end{array}$} \\
\hline & & $\lambda 8498$ & $\lambda 8542$ & $\lambda 8662$ & $\lambda 8498$ & $\lambda 8542$ & $\lambda 8662$ & $\lambda 8498$ & $\lambda 8542$ & $\lambda 8662$ \\
\hline HR 2269 & / 3 Cet & 0.84 & 0.78 & 2.14 & 2.45 & 3.25 & 1.67 & 2.06 & 2.54 & 3.58 \\
\hline HR 2269 & $\eta \quad$ Per & 1.12 & 1.20 & - & 2.3 & 2.3 & - & 2.58 & 2.75 & - \\
\hline $56 \mathrm{Peg}$ & $/ \zeta$ Cep & 1.50 & 1.15 & 1.23 & 2.36 & 3.25 & 3.05 & 3.54 & 3.74 & 3.74 \\
\hline $56 \mathrm{Peg}$ & $/ \epsilon \mathrm{Gem}$ & 1.06 & 1.56 & 1.54 & 2.6 & 2.16 & 2.65 & 2.77 & 3.38 & 4.08 \\
\hline$\beta$ Dra & $/ \zeta \mathrm{Gem}$ & 1.17 & 0.9 & 1.43 & 1.84 & 1.48 & 1.75 & 2.15 & 1.33 & 2.50 \\
\hline$\delta$ Vir & / $\eta$ Gem & 1.10 & 120 & 1.05 & 1.23 & 1.46 & 1.25 & 1.35 & 1.75 & 1.31 \\
\hline$\theta \mathrm{CMa}$ & $\alpha \quad \alpha \mathrm{Tau}$ & 0.70 & 0.69 & 0.63 & 1.52 & 2.10 & 1.98 & 1.06 & 1.45 & 1.25 \\
\hline$\nu$ Hya & $/ \beta \mathrm{Col}$ & 0.84 & 0.65 & - & 1.43 & 2.16 & - & 1.20 & 1.4 & - \\
\hline$\sigma$ Gem & $\alpha$ Gem & 0.83 & 0.71 & 0.81 & 1.64 & 2.47 & 2.17 & 1.36 & 1.75 & 1.76 \\
\hline$\lambda$ And & $/ \phi^{2}$ Ori & 0.8 & 0.76 & 0.88 & 1.90 & 2.37 & 1.87 & 1.52 & 1.80 & 1.58 \\
\hline$\epsilon$ Hya & / o UMa & 0.89 & 0.83 & 0.6 & 1.37 & 1.65 & 3.05 & 1.21 & 1.37 & 1.83 \\
\hline$\phi$ Vir & $\alpha$ Her & 0.77 & 0.69 & 0.67 & 1.37 & 2.27 & 1.88 & 1.05 & 1.57 & 1.25 \\
\hline$\phi \mathrm{Vir}$ & $/ \zeta$ Her & - & 0.6 & 0.7 & - & 1.54 & 1.82 & - & 0.92 & 1.27 \\
\hline$\epsilon$ Eri & $/ o^{2}$ Eri & 0.86 & 0.62 & 0.77 & 1.18 & 1.4 & 1.4 & 1.01 & 0.87 & 1.08 \\
\hline$\tau$ Cet & / 82 Eri & 0.82 & 0.63 & 0.50 & 1.75 & 1.52 & 1.60 & 1.44 & 0.96 & 0.80 \\
\hline$\chi^{1}$ Ori & $\beta$ Com & 0.92 & 0.64 & 1.05 & 1.27 & 1.71 & 1.4 & 1.17 & 1.09 & 1.47 \\
\hline$\chi^{1}$ Ori & $/ \beta \mathrm{Vir}$ & 0.86 & 0.85 & 1.10 & 1.32 & 1.83 & 1.42 & 1.14 & 1.56 & 1.57 \\
\hline HR 5317 & $/ \beta$ Cap & 1.25 & 1.00 & - & 1.87 & 2.62 & - & 2.34 & 2.62 & - \\
\hline
\end{tabular}

be chromospherically more active. Listed in the Cols. 5, 6 and 7 of Table 3, CRI is systematically higher in all the 3 lines in the first star of each pair for all pairs. For 4 of the chromospherically active stars, two examples each of above pairs are cited. The intensity index according to Wilson \& Bappu (1957) and Wilson (1976) in the more active stars is 3 or higher except for $\chi^{1}$ Ori as seen in Col. 3 of Table 3 . None of these stars are fast rotators. The quantity $v \sin i$ taken from the Bright Star Catalogue is listed in Col. 4. It is less than $20 \mathrm{~km} \mathrm{~s}^{-1}$ for most of the stars. Only $\beta$ Cap, $\zeta$ Gem and HR 5317 exceed this value. So rotational broadening cannot be affecting the CRI of the Ca II triplet lines in these stars. Since the latter star (i.e. $\beta$ Cap) in the pair HR 5317- $\beta$ Cap has higher $v \sin i$, the relatively higher CRI of HR 5317 cannot be due to rotational broadening. In fact, the Ca II lines of HR 5317 as seen in Fig. 7a seem a bit too weak for its high + ve $[\mathrm{Fe} / \mathrm{H}]$. We suspect that it is due to chromospheric emission filling in the star. $\tau$ Cet in Fig. $5 \mathrm{~b}$ has a rather low EQW perhaps again because chromospheric emission has partly filled in its absorption profile. As Cols. 8, 9 and 10 of Table 3 show, the EQWs of all the 3 lines are smaller in the star with the larger CRI than in the star with the smaller CRI. The amount by which they differ ranges from 10 to $30 \%$. However, this may not be the best way of measuring the chromospheric emission that fills in the absorption. Following the scheme of Linsky et al. (1979), we compared the Ca II profiles of each pair (stars with similar spectral type, luminosity \& metallicity) by dividing the spectrum of one with the larger CRI by that of the other with the smaller CRI point by point. Figure 9 shows some of these divided spectra in $\lambda \lambda 8498,8542$ and $\lambda 8662$.

These ratio profiles actually show net emission in each of the 3 lines, the amount that fills in the absorption profile of the star having larger CRI, i.e., with larger chromospheric activity. They also show the spectral range over which the chromospheric filling in occurs in the active chromosphere stars. Columns 2, 3 and 4 of Table 4 list the FWHM of the ratio profiles of the 3 lines in the 13 star pairs given in Col. 1. It would be interesting to see how the FWHM relates to the luminosity of the stars in question. The ratio plots give FWHM for the 3 lines in supergiants in the range $0.9 \AA-1.6 \AA$, in giants from $0.7 \AA$ to $1.1 \AA$ and in dwarfs \& subgiants from $0.6 \AA$ to $0.9 \AA$. The FWHM does increase with luminosity, quite in analogy with the behaviour of the Ca II H and K line emission widths to luminosity. The peak values of the emission profiles of all the 3 lines are displayed in Cols. 5, 6, and 7 of Table 4. The product of FWHM and the peak value of emission (which is actually a more direct measure of the amount of emission) was calculated for all the dwarf, giant and supergiant pairs and is shown in Cols. 8, 9 and 10. We find that this quantity is more sensitive to luminosity than FWHM alone. In supergiants, it lies in the range $2.1-4.0$ for the 3 lines, in giants between $1.1-1.8$ and in dwarfs $\&$ subgiants between $0.9-1.5$. Most of the ratio profiles differ appreciably from unity only in the line core (within $\pm 1 \AA$ of the line core) implying substantial emission in the chromosphere but not in the photosphere. According to Linsky et al. (1979), a better way of detecting photospheric emission apart from the chromospheric emission, 

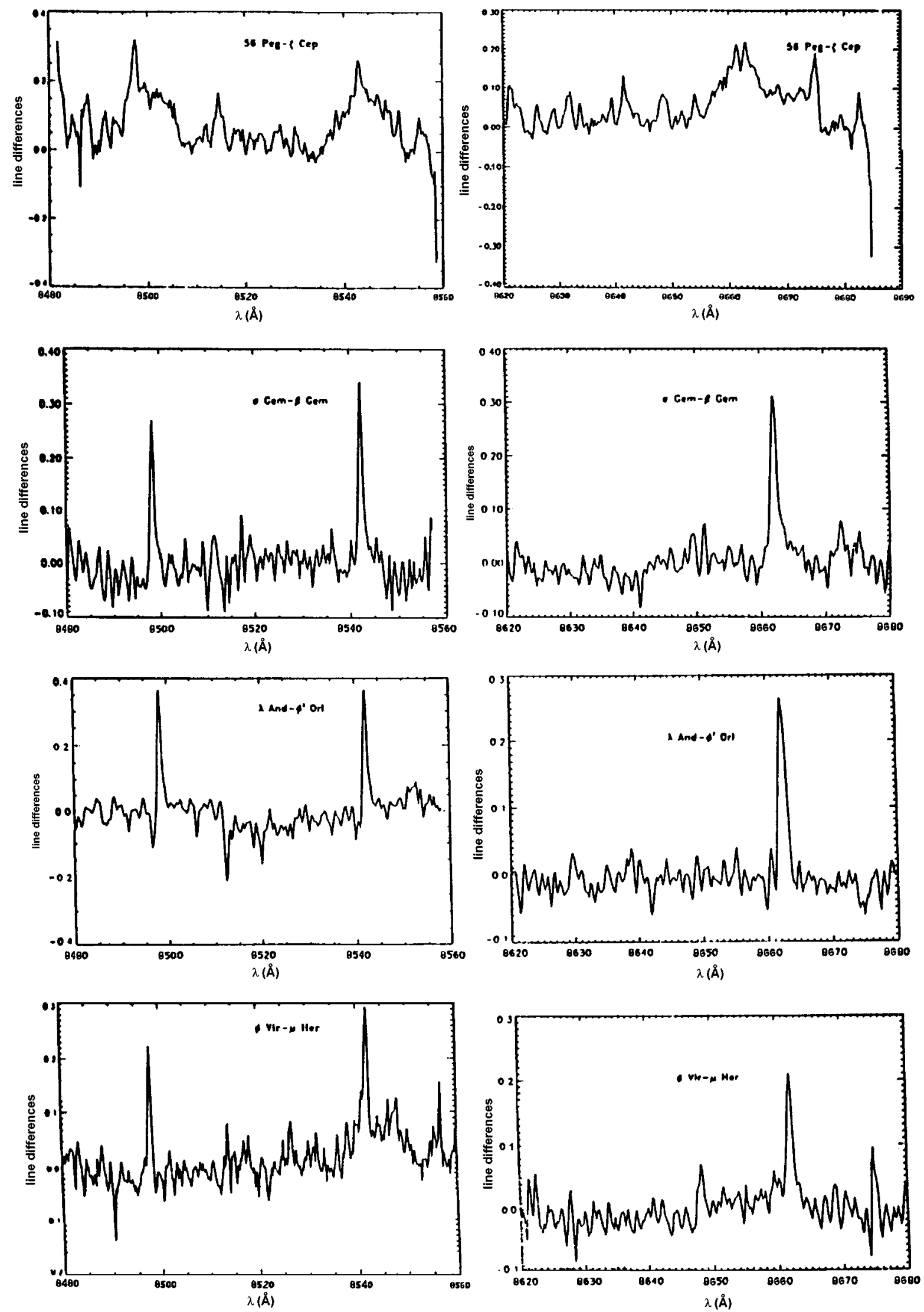

Fig. 10. Line difference spectra of $\lambda \lambda 8498,8542,8662$ of the same pairs 
is to subtract the profiles of similar stars. Figure 10 shows the line difference plots for $\lambda \lambda 8498,8542$ and $\lambda 8662$ for a few star pairs. The pairs 56 Peg- $\zeta$ Cep, 56 Peg- $\epsilon$ Gem, HR 2269-3 Cet, HR 2269- $\eta$ Per are much more spread out (out to more than $\pm 3 \AA$ of the line core) than the other pairs, suggesting a strong evidence of emission beyond the line core, implying it originates in the photosphere.

Thus, if the CRI of the Ca II triplet lines is not affected by parameters like rotational broadening, macroturbulence, microturbulence etc., then the CRI of especially the $8542 \AA$ and the $8662 \AA$ lines could be viewed as a diagnostic and a measure of chromospheric activity in stars. The nearly one-to-one correlation between FWHM/area of the ratio profiles and the Ca II K line emission intensity index supports the above inference. Linsky et al. (1979) also strongly support the contention that a direct comparison of $\lambda 8542$ CRIs of stars with similar spectral type and luminosity class should at least be a qualitative discriminant of chromospheric activity. The direct spectroscopic diagnostic is the chromospheric radiative loss rate which is known to be significantly higher in active chromosphere stars than in quiet chromosphere stars. Linsky et al. (1979) have estimated the $\lambda 8542$ chromospheric radiative loss rate in stars they have observed and find that stars with larger $\lambda 8542$ chromospheric radiative loss rates are also those with larger Ca II K line intensities. Since the $\lambda 8542$ CRI's of the stars in each group of stars with similar spectral and luminosity type have one-to-one correlation with the K-line index, we contend that $\lambda 8542 \mathrm{CRI}$ is a good discriminant of chromospheric activity. Cayrel de Strobel (1992) has observed the Ca II $\lambda \lambda 8498,8542$ lines in a small sample of F, G and K dwarfs and slightly evolved subgiants randomly selected from the "Catalogue of Nearby Stars" of Gliese (1969). Observed profiles of these lines in 6 early $\mathrm{K}$ dwarfs having about the same effective temperature show that the shallower the lines are in the spectrum of a star, the more active the chromosphere of this star is.

\section{Conclusions}

We have observed the Ca II triplet lines in 146 stars to understand the interplay of such parameters as temperature, gravity and chemical composition in determining the strengths of these lines. Our observations span all luminosity types, a range in spectral types from F8 to M4 and in $[\mathrm{Fe} / \mathrm{H}]$ from -3.0 to +1.1 . The detailed empirical analysis has stressed on the importance of considering a sufficient range in $T_{\text {eff }}, \log g$ and $[\mathrm{Fe} / \mathrm{H}]$ to cover all stars that contribute to the integrated light of the calcium lines in a stellar system and has shown a more complex dependence of the EQW of the Ca II lines on the above parameters than previously found. The salient conclusions are:

a) The Ca II triplet EQWs are indeed sensitive to surface gravity. Assuming it to be a single valued linear relation, the observers in the past have used it to determine the luminosity of stars in galaxies. However, this relationship is nonlinear over the $\log g$ range covered and is also influenced by $T_{\text {eff }}$ and $[\mathrm{Fe} / \mathrm{H}]$ in complicated ways.

b) The dependence of the EQW on surface gravity is steeper for metal rich stars than for metal poor stars. This fact can be used to determine the luminosity of the dominant population from the Ca II spectra in high metallicity composite stellar systems. It is not possible to derive useful information from the Ca II spectra in low metallicity stellar systems unless the effective temperature of the stars contributing to the light is known independently.

c) The Ca II triplet EQWs are only mildly dependent on effective temperature. And even here, giants and dwarfs respond differently to changes in $T_{\text {eff }}$.

d) Dwarfs and giants respond differently even to metallicity. The sensitivity of Ca II EQW on metallicity is strong for supergiants and weaker in giants and dwarfs, even though the range in $[\mathrm{Fe} / \mathrm{H}]$ covered in the latter is much larger. Even with $[\mathrm{Fe} / \mathrm{H}]$ ranging only from -0.88 to +0.50 in supergiants, the correlation appears to be nonlinear.

e) It is found that stars of the same temperature, luminosity and metallicity may have varying central depths and this has to do with the chromospheric activity varying among these stars. The shallower the lines in the spectrum of a star, the more active the chromosphere of this star is. Chromospheric emission fills in the absorption profile and raises the core.

The Ca II triplet line strength and shape thus depend not only on luminosity and metallicity but also on chromospheric activity. To be able to use the Ca II triplet lines as a tool for discriminating stellar populations in galaxies, one has to understand the complex behaviour of the Ca II triplet lines with respect to all these parameters. Only when the sensitivity to each parameter is independently determined, can one exploit this information in the understanding of the stellar content of galaxies. The Ca II triplet lines as a potential probe for the stellar population synthesis should be viewed in the light of this complex dependence.

Acknowledgements. I especially wish to thank the referee for several useful comments and suggestions that have helped improve paper.

\section{References}

Allen C.W., 1973, Astronomical Quantities, Third edition, University of London. The Althone press, p. 213

Alloin D., Bica E., 1989, A\&A 217, 57

Anderson C.M., 1974, ApJ 190, 585

Bell R.A., Gustaffson B., 1989, MNRAS 236, 653

Bohm-Vitense E., 1972, A\&A 17, 335

Carter D., Visvanathan N., Pickles A.J., 1986, ApJ 311, 637

Cassinelli J.P., MacGregor K.B., 1986, Physics of the Sun, Vol. III, Sturrock P.A. (ed.). D. Reidel, p. 47

Cayrel de Strobel G., 1992, The ESO Messenger No. 70, West R.M. (ed.) p. 37 
Cayrel de Strobel G., Hauck B., Francois P., et al., 1992, A\&AS 95, 273

Diaz A.I., Terlevich E., Terlevich R., 1989, MNRAS 239, 325 (DTT)

Erdelyi-Mendes M., Barbuy B., 1991, A\&A 241, 176

Flower P.J., 1977, A\&A 54, 31

Gliese W., 1969, Catalogue of nearby stars, Veroff. Astron. Rechen-Inst. Heidelberg, No. 22

Hoffleit D., 1982, The Bright Star Catalogue, Fourth revised edition, Yale University Observatory, New Haven, Connecticut

Horne K., 1986, PASP 98, 609

Horne K., 1988, New Directions in Spectrophotometry, Philip A.G.D., Hayes D., Adelman S. (eds.). Schenactedy: L. Davies Press

Jones J.E., Alloin D.M., Jones B.J.T., 1984, ApJ 283, 457 (JAJ)

Jorgenson U.G., Carlsson M., Johnson H.R., 1992, A\&A 254, 258 (JCJ)
Keenan P.C., McNeil R.C., 1976, An Atlas of Spectra of the Cooler Stars: Types G, K, M, S, and C. The Ohio State University Press

Lang K.R., 1992, Astrophsical Data: Planets and Stars. Springer-Verlag, p. 145

Linsky J.L., 1980, ARA\&A 18, 439

Linsky J.L., Hunten D.M., Sowell R., Glackin D.L., Kelch W.L., 1979, ApJS 41, 481

Luck R.E., Bond H.E., 1980, ApJ 241, 218

Mallik S.V., 1994, A\&AS 103, 279

Prabhu T.P., Anupama G.C., 1991, Bull. ASI 19, 97

Schmidt-Kaler Th., 1982, Landolt-Bornstein series, Group VI, Vol. 2b, Schaifers K., Voigt H.H. (eds.). Springer-Verlag, p. 451

Smith G., Drake J.J., 1987, A\&A 181, 103

Smith G., Drake J.J., 1990, A\&A 231, 125

Wilson O.C., 1976, ApJ 205, 823

Wilson O.C., Bappu M.K.V., 1957, ApJ 125, 661

Zhou X., 1991, A\&A 248, 367 